\title{
Reusable Copper-Catalyzed Cross- Coupling Reactions of Aryl Halides with Organotins in Inexpensive Ionic Liquids
}

Jin-Heng Li, * Bo-Xiao Tang, Li-Ming Tao, Ye-Xiang Xie, Yun Liang, and Man-Bo Zhang*

Key Laboratory of Chemical Biology \& Traditional Chinese Medicine Research (Ministry of Education), College of Chemistry and Chemical Engineering, Hunan Normal University, Changsha 410081, China

\section{Supporting Information}

\section{List of Contents}
(A) Remarks
(B) Typical experimental procedure
(C) Analytical data for 3-13
S2-5
(D) References
S5-6
(E) Synthesis of $\mathrm{Cu}_{2} \mathrm{O}$ Nanoparticles
S6-9
(F) Spectra
S10-31 


\section{(A) Remarks:}

NMR spectroscopy was performed on an INOVA-400 (Varian), an INOVA-500 (Varian) or an AMX-300 (Bruker) spectrometer operating at $400 \mathrm{MHz}\left({ }^{1} \mathrm{H} \mathrm{NMR}\right)$ and $100 \mathrm{MHz}\left({ }^{13} \mathrm{C}\right.$ NMR $), 500 \mathrm{MHz}\left({ }^{1} \mathrm{H} \mathrm{NMR}\right)$ and $125 \mathrm{MHz}\left({ }^{13} \mathrm{C} \mathrm{NMR}\right)$, or $300 \mathrm{MHz}\left({ }^{1} \mathrm{H}\right.$ NMR) and $75 \mathrm{MHz}\left({ }^{13} \mathrm{C}\right.$ NMR). TMS (tetramethylsilane) was used an internal standard and $\mathrm{CDCl}_{3}$ was used as the solvent.

\section{(B) Typical experimental procedure}

Typical Experimental Procedure for the Copper-Catalyzed Stille cross-coupling reaction in TBAB. A mixture of aryl halide $1(0.3 \mathrm{mmol})$, organotin $2(0.4 \mathrm{mmol}), \mathrm{Cu}_{2} \mathrm{O}$ (nanoparticles; $10 \mathrm{~mol} \%), \mathrm{P}(o \text {-tol })_{3}\left(\mathbf{L}_{7} ; 6 \mathrm{~mol} \%\right), \mathrm{KF} \cdot 2 \mathrm{H}_{2} \mathrm{O}$ (2 equiv) and TBAB (1.5 g) was stirred at $125-130{ }^{\circ} \mathrm{C}$ for desired time until complete consumption of starting material as monitored by TLC. After the reaction was finished, diethyl ether was poured into the mixture, then washed with water, extracted with diethyl ether, dried by anhydrous $\mathrm{Na}_{2} \mathrm{SO}_{4}$ and evaporated under vacuum, the residue was purified by flash column chromatography (hexane or hexane/ethyl acetate) to afford the desired coupled product.

After initial experimentation, the residue $\mathrm{Cu}_{2} \mathrm{O}$ nanoparticles $/ \mathrm{P}(o \text {-tol })_{3} / \mathrm{TBAB}$ system $)$ were then solidified (evaporated in vacuo and cooled) and subjected to a second run of the Stille reaction by charging with the same substrates (aryl halide, organotin and $\left.\mathrm{KF} \cdot 2 \mathrm{H}_{2} \mathrm{O}\right)$.

\section{(C) Analytical data for 3-13}




\section{4-Nitro-biphenyl (3) ${ }^{1}$}

Yellow solid; ${ }^{1} \mathrm{H}$ NMR $\left(500 \mathrm{MHz}, \mathrm{CDCl}_{3}\right) \delta: 8.32(\mathrm{~d}, J=8.8 \mathrm{~Hz}, 2 \mathrm{H}), 7.76(\mathrm{~d}, J=8.8$ $\mathrm{Hz}, 2 \mathrm{H}), 7.65(\mathrm{~d}, J=6.9 \mathrm{~Hz}, 2 \mathrm{H}), 7.55-7.48(\mathrm{~m}, 3 \mathrm{H}) ;{ }^{13} \mathrm{C} \mathrm{NMR}\left(125 \mathrm{MHz}, \mathrm{CDCl}_{3}\right) \delta$ : 147.6, 147.1, 138.8, 129.2, 128.9, 127.8, 127.4, 124.1. LRMS (EI, $20 \mathrm{eV}) \mathrm{m} / \mathrm{z}(\%): 199$ $\left(\mathrm{M}^{+}, 100\right)$.

\section{1-Nitro-4-vinylbenzene $(4)^{2}$}

Yellow oil; ${ }^{1} \mathrm{H}$ NMR (400 MHz, $\left.\mathrm{CDCl}_{3}\right) \delta: 8.19(\mathrm{~d}, J=9.2 \mathrm{~Hz}, 2 \mathrm{H}), 7.54(\mathrm{~d}, J=8.8$ $\mathrm{Hz}, 2 \mathrm{H}), 6.81,6.76(\mathrm{dd}, J=10.8 \mathrm{~Hz}, 10.8 \mathrm{~Hz}, 1 \mathrm{H}), 5.94$ (d, $J=17.6 \mathrm{~Hz}, 1 \mathrm{H}), 5.50$ (d, $J=$ $11.2 \mathrm{~Hz}, 1 \mathrm{H}) ;{ }^{13} \mathrm{C}$ NMR $\left(100 \mathrm{MHz}, \mathrm{CDCl}_{3}\right) \delta: 147.2,143.8,135.0,126.8,123.9,118.6$. LRMS (EI, $20 \mathrm{eV}) m / z(\%): 149\left(\mathrm{M}^{+}, 100\right)$.

\section{1-(2-(4-Nitrophenyl)ethynyl)benzene (5)}

slight yellow solid; ${ }^{1} \mathrm{H}$ NMR $\left(500 \mathrm{MHz}, \mathrm{CDCl}_{3}\right) \delta: 8.25(\mathrm{~d}, J=8.8 \mathrm{~Hz}, 2 \mathrm{H}), 7.60(\mathrm{~d}, J$ $=8.8 \mathrm{~Hz}, 2 \mathrm{H}), 7.60-7.58(\mathrm{~m}, 2 \mathrm{H}), 7.43-7.41(\mathrm{~m}, 3 \mathrm{H}) ;{ }^{13} \mathrm{C} \mathrm{NMR}\left(125 \mathrm{MHz}, \mathrm{CDCl}_{3}\right) \delta$ : 147.1, 132.2, 131.8, 130.3, 129.3, 128.5, 123.6, 122.2, 94.7, 87.5. LRMS (EI, $20 \mathrm{eV}) \mathrm{m} / \mathrm{z}$ (\%): $223\left(\mathrm{M}^{+}, 100\right)$.

\section{1-Biphenyl-4-yl-ethanone (6) ${ }^{1}$}

White solid; ${ }^{1} \mathrm{H}$ NMR $\left(300 \mathrm{MHz}, \mathrm{CDCl}_{3}\right) \delta: 8.04(\mathrm{~d}, J=8.4 \mathrm{~Hz}, 2 \mathrm{H}), 7.69$ (d, $J=8.4$ $\mathrm{Hz}, 2 \mathrm{H}), 7.64(\mathrm{~d}, J=7.6 \mathrm{~Hz}, 2 \mathrm{H}), 7.50-7.40(\mathrm{~m}, 3 \mathrm{H}), 2.64(\mathrm{~m}, 3 \mathrm{H}),{ }^{13} \mathrm{C} \mathrm{NMR}(75 \mathrm{MHz}$, $\left.\mathrm{CDCl}_{3}\right): \delta 197.8,145.8,139.9,135.8,128.9,128.9,128.2,127.3,127.2,26.7$. LRMS (EI, $20 \mathrm{eV}) \mathrm{m} / z(\%): 196\left(\mathrm{M}^{+}, 100\right)$. 


\section{4-Methoxy-biphenyl (7) ${ }^{1}$}

White solid; ${ }^{1} \mathrm{H}$ NMR (500 MHz, $\left.\mathrm{CDCl}_{3}\right) \delta: 7.60-7.56(\mathrm{~m}, 4 \mathrm{H}), 7.46$ (t, $J=7.8 \mathrm{~Hz}$, 2H), $7.34(\mathrm{t}, J=7.5,1 \mathrm{H}), 7.02(\mathrm{~d}, J=9.0,2 \mathrm{H}), 3.89(\mathrm{~s}, 3 \mathrm{H}) ;{ }^{13} \mathrm{C} \mathrm{NMR}\left(125 \mathrm{MHz}, \mathrm{CDCl}_{3}\right)$ $\delta: 159.2,140.9,133.8,128.7,128.2,126.8,126.7,114.2,55.4$. LRMS (EI, $20 \mathrm{eV}) \mathrm{m} / \mathrm{z}$ (\%): $184\left(\mathrm{M}^{+}, 100\right)$.

\section{1-(2-(4-Methoxyphenyl)ethynyl)benzene (8) ${ }^{3}$}

White solid; ${ }^{1} \mathrm{H}$ NMR (400 MHz, $\left.\mathrm{CDCl}_{3}\right) \delta: 7.52-7.51(\mathrm{~m}, 2 \mathrm{H}), 7.48(\mathrm{~d}, J=9.0 \mathrm{~Hz}$, 2H), 7.34-7.32 (m, 3H), $6.88(\mathrm{~d}, J=8.8 \mathrm{~Hz}, 2 \mathrm{H}), 3.83(\mathrm{~s}, 3 \mathrm{H}) ;{ }^{13} \mathrm{C}$ NMR (100 MHz, $\left.\mathrm{CDCl}_{3}\right) \delta: 159.6,133.0,131.4,128.3,127.9,123.6,115.4,114.0,89.4,88.1,55.3 ;$ LRMS (EI, $20 \mathrm{eV}) m / z(\%): 208\left(\mathrm{M}^{+}, 100\right)$.

\section{1-(4-(2-Phenylethynyl)phenyl)ethanone (9)}

White solid; ${ }^{1} \mathrm{H}$ NMR (400 MHz, $\left.\mathrm{CDCl}_{3}\right) \delta: 7.95(\mathrm{~d}, J=8.8 \mathrm{~Hz}, 2 \mathrm{H}), 7.61(\mathrm{~d}, J=8.8$ $\mathrm{Hz}, 2 \mathrm{H}), 7.57-7.55(\mathrm{~m}, 2 \mathrm{H}), 7.38(\mathrm{t}, J=3.2 \mathrm{~Hz}, 3 \mathrm{H}), 2.63(\mathrm{~s}, 3 \mathrm{H}) ;{ }^{13} \mathrm{C}$ NMR $(100 \mathrm{MHz}$, $\left.\mathrm{CDCl}_{3}\right) \delta: 197.4,136.1,131.8,131.7,128.8,128.4,128.3,122.6,92.7,88.6,26.7$; LRMS (EI, $20 \mathrm{eV}) m / z(\%): 220\left(\mathrm{M}^{+}, 100\right)$.

\section{Biphenyl (10)}

White solid; ${ }^{1} \mathrm{H}$ NMR $\left(300 \mathrm{MHz}, \mathrm{CDCl}_{3}\right) \delta: 7.59(\mathrm{~d}, J=8.4 \mathrm{~Hz}, 4 \mathrm{H}), 7.43(\mathrm{t}, J=7.2$ $\mathrm{Hz}, 4 \mathrm{H}), 7.36(\mathrm{t}, J=7.8 \mathrm{~Hz}, 2 \mathrm{H}) ;{ }^{13} \mathrm{C} \mathrm{NMR}\left(75 \mathrm{MHz}, \mathrm{CDCl}_{3}\right) \delta: 141.6,129.1,127.6$, 127.5. LRMS (EI, $20 \mathrm{eV}) \mathrm{m} / z(\%): 154\left(\mathrm{M}^{+}, 100\right)$. 


\section{1,2-Diphenylethyne (11) ${ }^{3}$}

White solid; ${ }^{1} \mathrm{H}$ NMR (300 MHz, $\left.\mathrm{CDCl}_{3}\right) \delta: 7.60-7.51(\mathrm{~m}, 4 \mathrm{H}), 7.39-7.26(\mathrm{~m}, 6 \mathrm{H}) ;{ }^{13} \mathrm{C}$ NMR (75 MHz, $\mathrm{CDCl}_{3}$ ) $\delta: 132.0,128.7,128.6,123.7,89.7$; LRMS (EI, $\left.20 \mathrm{eV}\right) \mathrm{m} / z(\%)$ : $178\left(\mathrm{M}^{+}, 100\right)$.

\section{4-Methyl-biphenyl (12) ${ }^{1}$}

White solid; ${ }^{1} \mathrm{H}$ NMR $\left(400 \mathrm{MHz}, \mathrm{CDCl}_{3}\right) \delta: 7.58(\mathrm{t}, J=7.6 \mathrm{~Hz}, 2 \mathrm{H}), 7.49$ (d, $J=8.0$ $\mathrm{Hz}, 2 \mathrm{H}), 7.42(\mathrm{t}, \mathrm{J}=7.6 \mathrm{~Hz}, 2 \mathrm{H}), 7.31(\mathrm{t}, J=7.6 \mathrm{~Hz}, 1 \mathrm{H}), 7.24(\mathrm{~d}, J=8.0 \mathrm{~Hz}, 2 \mathrm{H}), 2.38(\mathrm{~s}$, $3 \mathrm{H}) ;{ }^{13} \mathrm{C} \mathrm{NMR}\left(100 \mathrm{MHz}, \mathrm{CDCl}_{3}\right) \delta: 141.1,138.3,137.0,129.5,128.7,127.3,127.2$, 127.0, 21.1. LRMS (EI, $20 \mathrm{eV}) \mathrm{m} / z(\%): 168\left(\mathrm{M}^{+}, 100\right)$.

\section{3,5-Dimethyl-biphenyl (13) ${ }^{5}$}

Colorless oil; ${ }^{1} \mathrm{H}$ NMR (400 MHz, $\left.\mathrm{CDCl}_{3}\right) \delta: 7.59$ (d, $\left.J=8.4 \mathrm{~Hz}, 2 \mathrm{H}\right), 7.44-7.40$ (m, 2H), 7.31-7.28 (m, 1H), $7.19(\mathrm{~d}, J=8.4 \mathrm{~Hz}, 2 \mathrm{H}), 6.98(\mathrm{~d}, J=9.2 \mathrm{~Hz}, 1 \mathrm{H}), 2.35(\mathrm{~s}, 3 \mathrm{H})$, 2.37 (s, 3H); ${ }^{13} \mathrm{C}$ NMR (100 MHz, $\left.\mathrm{CDCl}_{3}\right) \delta: 141.5,138.1,128.9,128.7,127.9,127.2$, 127.1, 125.1, 21.4. LRMS (EI, $20 \mathrm{eV}) m / z(\%): 182\left(\mathrm{M}^{+}, 100\right)$.

\section{(D) References}

(1) (a) Liu, D.; Gao, W.; Dai, Q. Zhang, X. Org. Lett. 2005, 7, 4907. (b) Thathagar, M. B. Beckers, J.; Rothenberg, G. Adv. Synth. Chem. 2003, 345, 979.

(2) Broos, R.; Anteunis, M. Synth. Commun. 1976, 6, 53.

(3) (a) Ma, D, Liu, F. Chem. Commun. 2004, 1934. (b) Okuro, K.; Furuune, M.; Enna, M.; Miura, M.; Nomura, M. J. Org. Chem. 1993, 58, 4716. (c) Gujadhur, R. K.; 
Bates, C. G.; Venkataraman, D. Org. Lett. 2001, 3, 4315. (d) Saejueng, P.; Bates, C. G.; Venkataraman, D. Synthesis. 2005, 1706. (e) Yi, C.; Hua, R. J. Org. Chem. 2006, $71,2535$.

(4) Venkatraman, S.; Li, C.-J. Org. Lett. 1999, 1, 1133.

(5) Akiyama, R.; Kobayashi, S. Angew. Chem. Int. Ed. 2001, 40, 3469.

\section{(E) Synthesis of $\mathrm{Cu}_{2} \mathrm{O}$ Nanoparticles ${ }^{6}$}

Experimental Procedure for the Synthesis of $\mathrm{Cu}_{2} \mathrm{O}$ Nanoparticles. A mixture of PEG$6000(300 \mathrm{mg}), \mathrm{Cu}(\mathrm{OAc})_{2} \cdot \mathrm{H}_{2} \mathrm{O}(399 \mathrm{mg})$ and $\mathrm{D}-(+)$-glucose $(1800 \mathrm{mg})$ was dissolved in water $(100 \mathrm{~mL})$. The mixture was stirred at $50{ }^{\circ} \mathrm{C}$ to ensure them dissolved completely, followed by dropwise addition of $\mathrm{NaOH}(5 \mathrm{M}, 2 \mathrm{~mL})$ into the solution. After the solution was stirred at $50{ }^{\circ} \mathrm{C}$ for $40 \mathrm{~min}$, the $\mathrm{Cu}_{2} \mathrm{O}$ precipitate was isolated by centrifuge, then washed with distilled water, ethanol and acetone in turn, and dried in a vacuum oven at $40{ }^{\circ} \mathrm{C}$ to offer $\mathrm{Cu}_{2} \mathrm{O}$ nanoparticles $(110 \mathrm{mg})$.

(6) Wang, W.; Wang, G.; Wang, X.; Zhan, Y.; Liu, Y.; Zheng, C. Adv. Mater. 2002, 14, 67. 


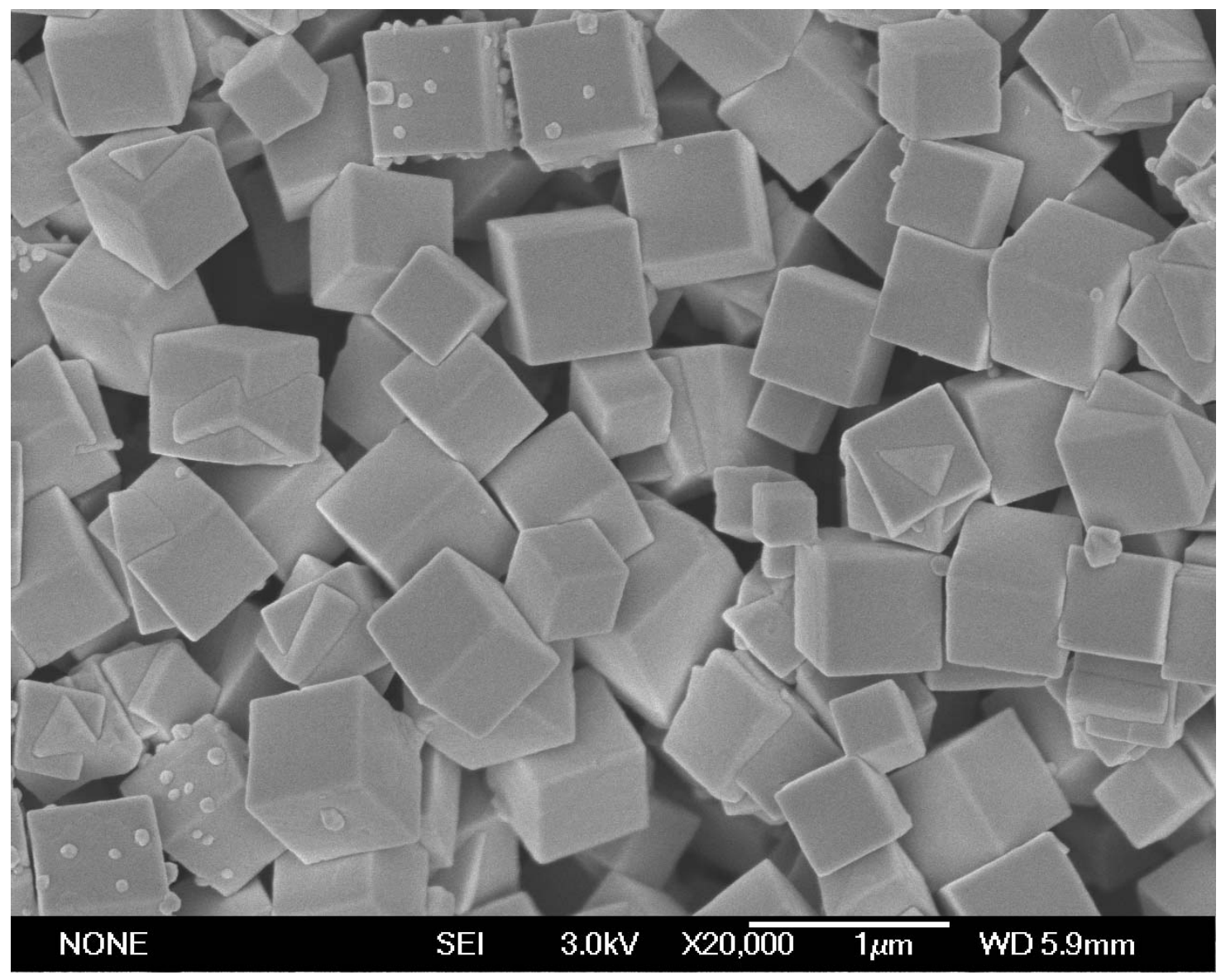

Figure 1. TEM picture showing $\mathrm{Cu}_{2} \mathrm{O}$ nanoparticles.

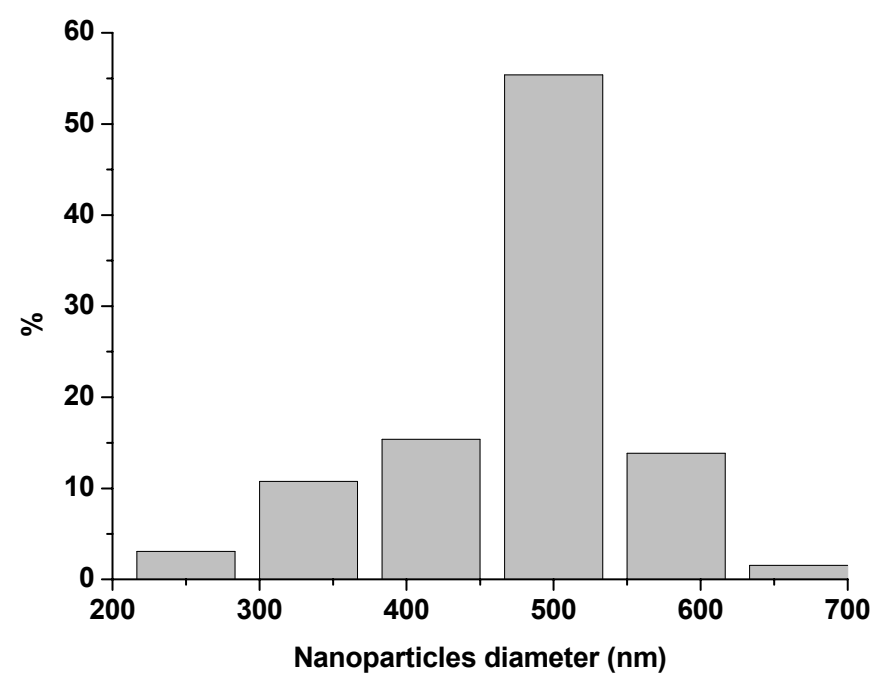

Figure 2. Histogram of the $\mathrm{Cu}_{2} \mathrm{O}$ nanoparticles' size distribution 
Figure 3. XRD pattern of the $\mathrm{Cu}_{2} \mathrm{O}$ nanoparticles

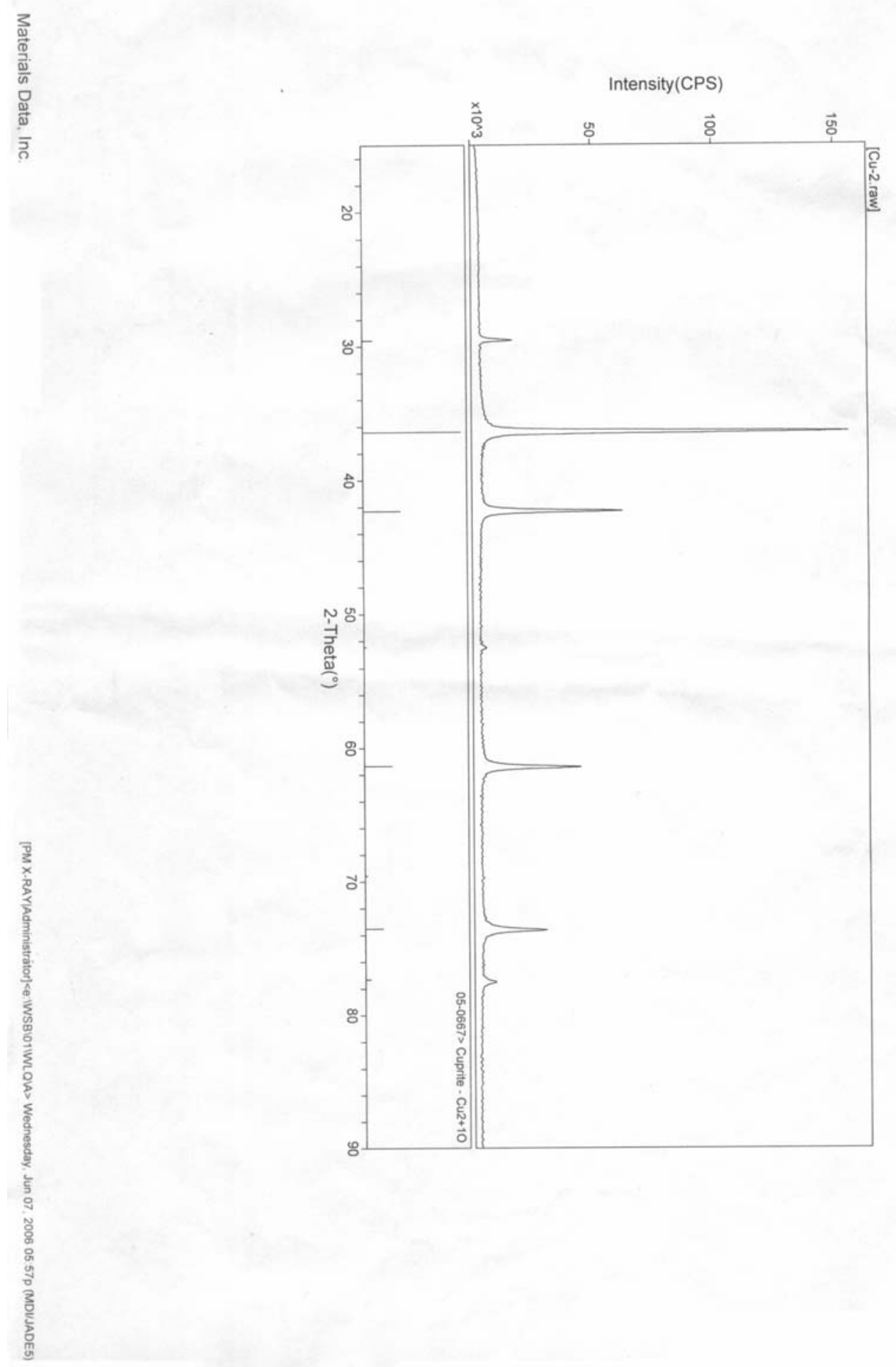




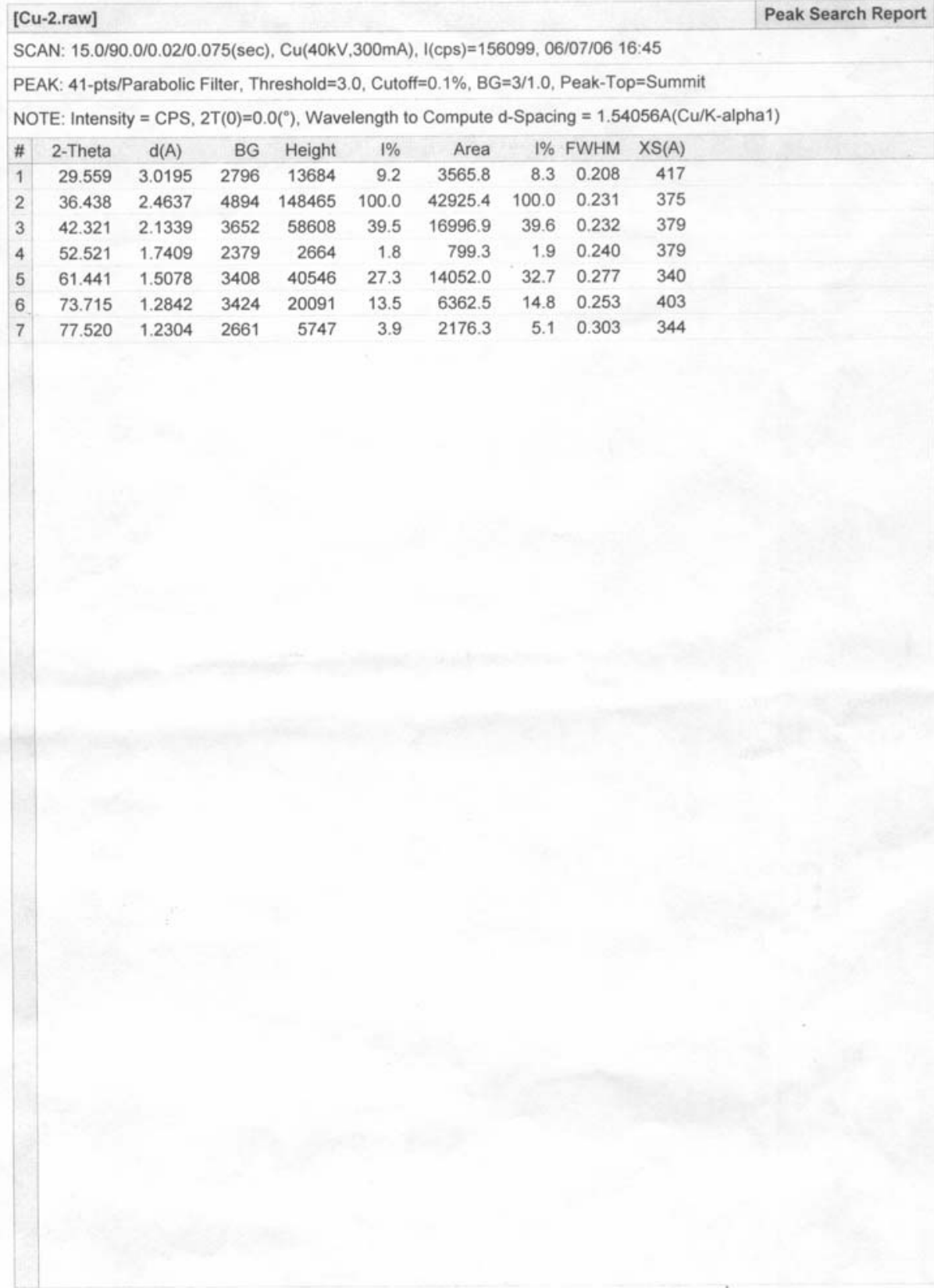


(F) Spectra

4-Nitro-biphenyl (3)

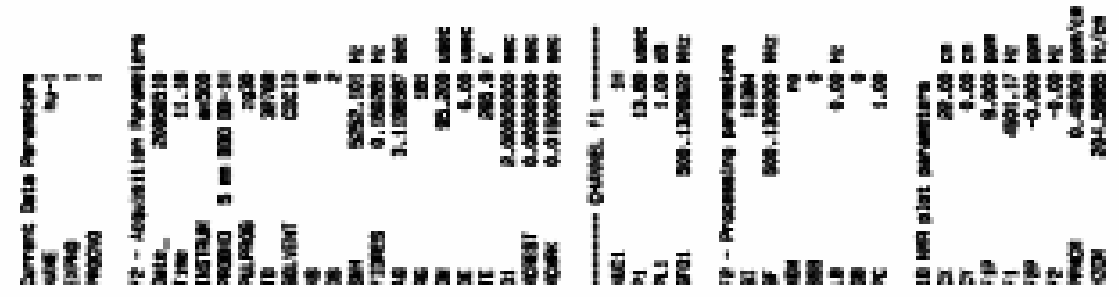

staeg'1-
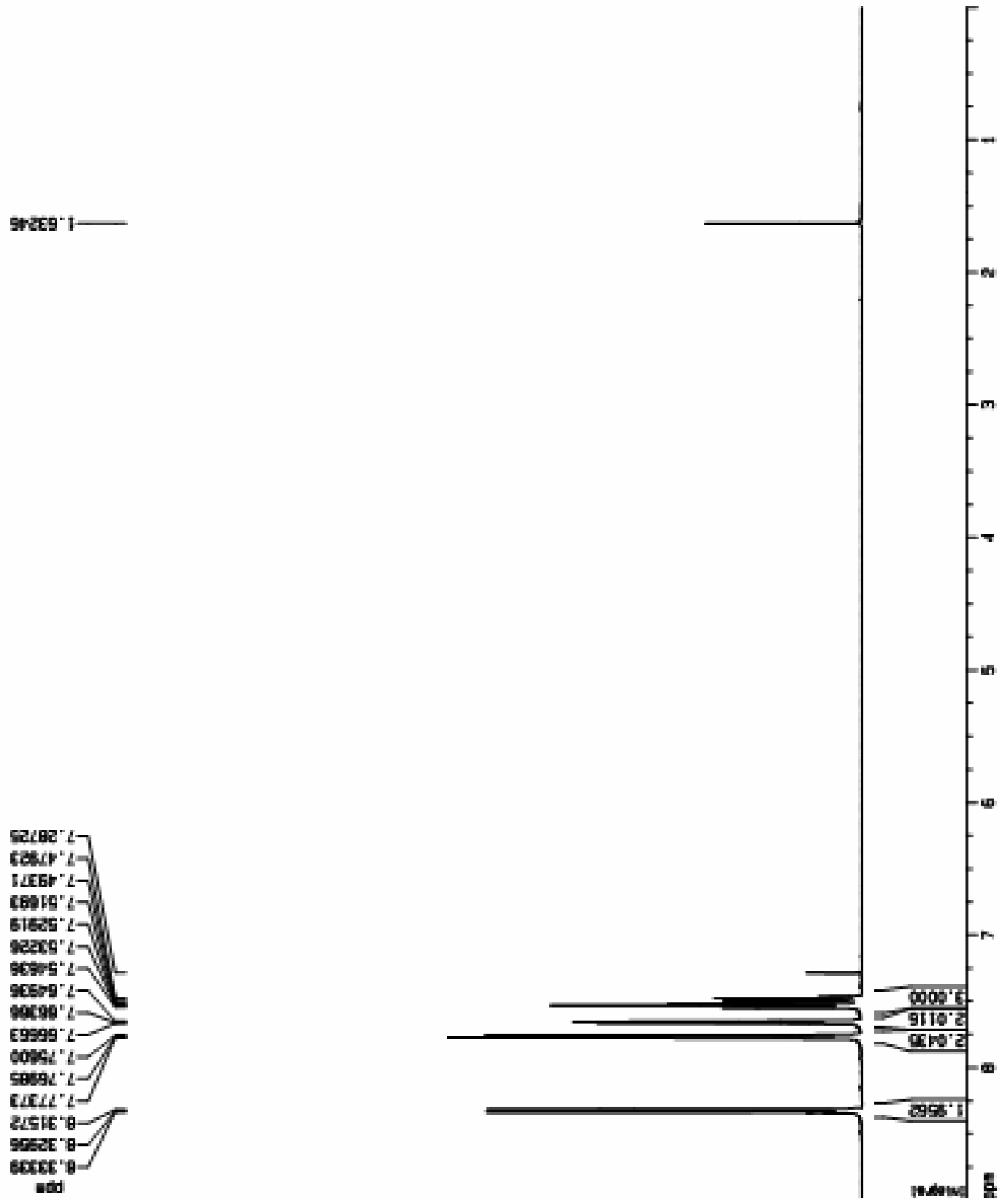
4-Nitro-biphenyl (3)

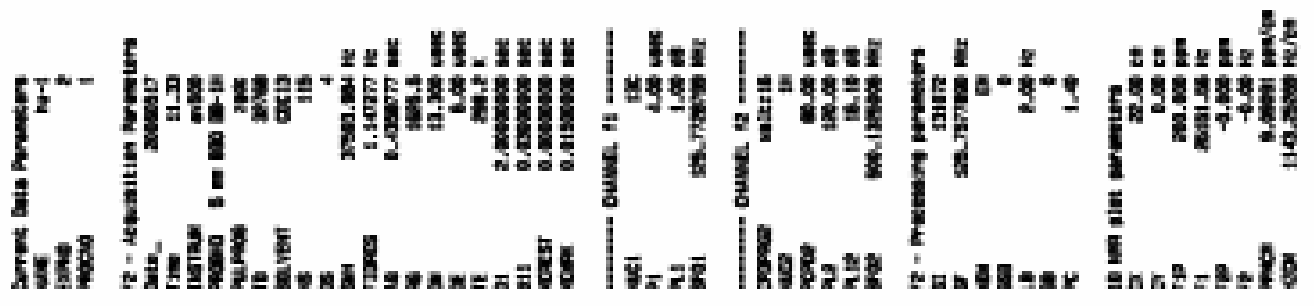

I

$790 \cdot L$

ตा' 2

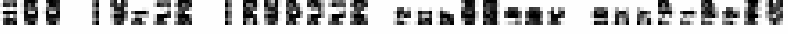

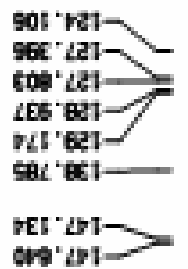

atd

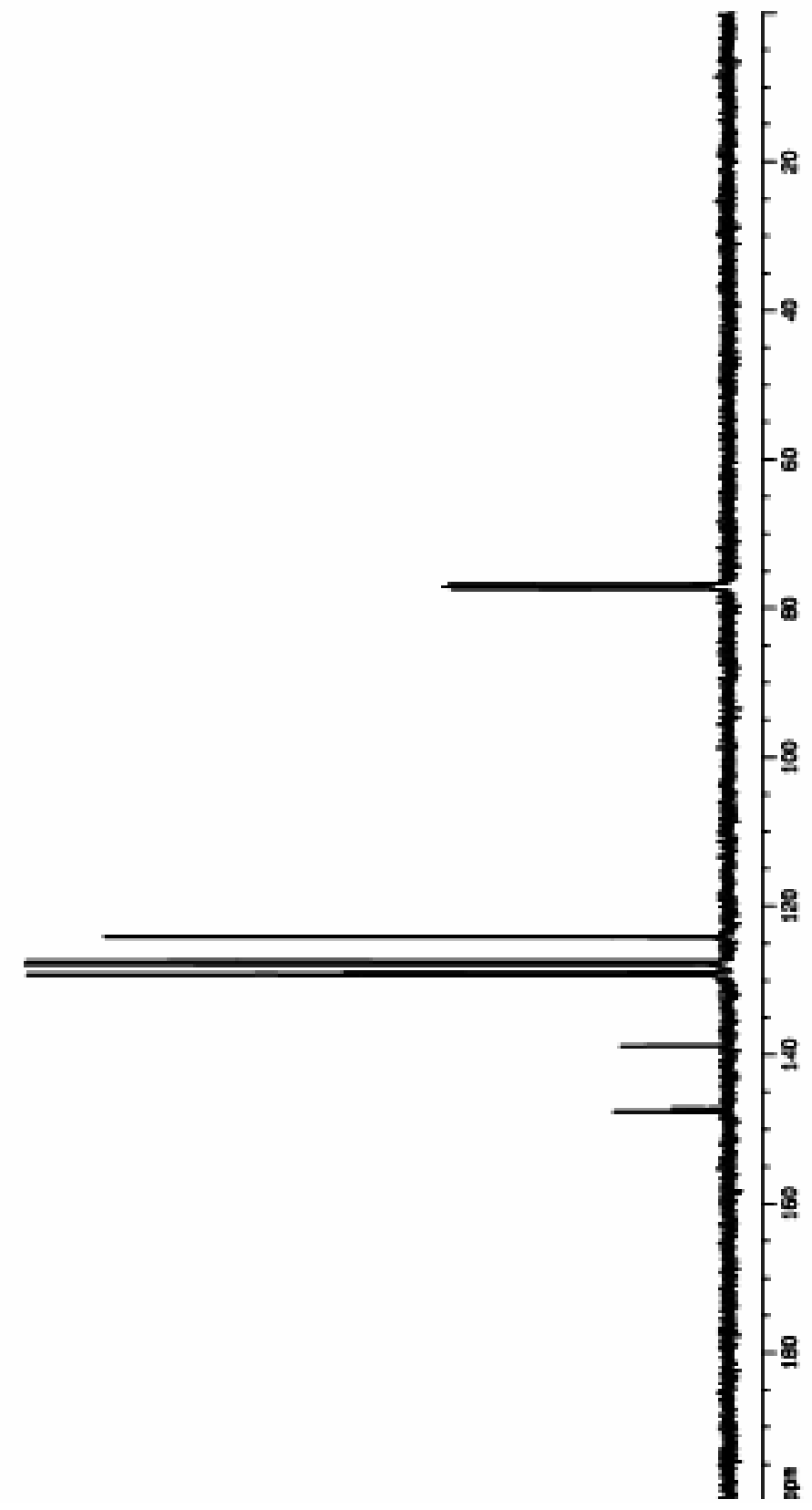


1-Nitro-4-vinylbenzene (4)
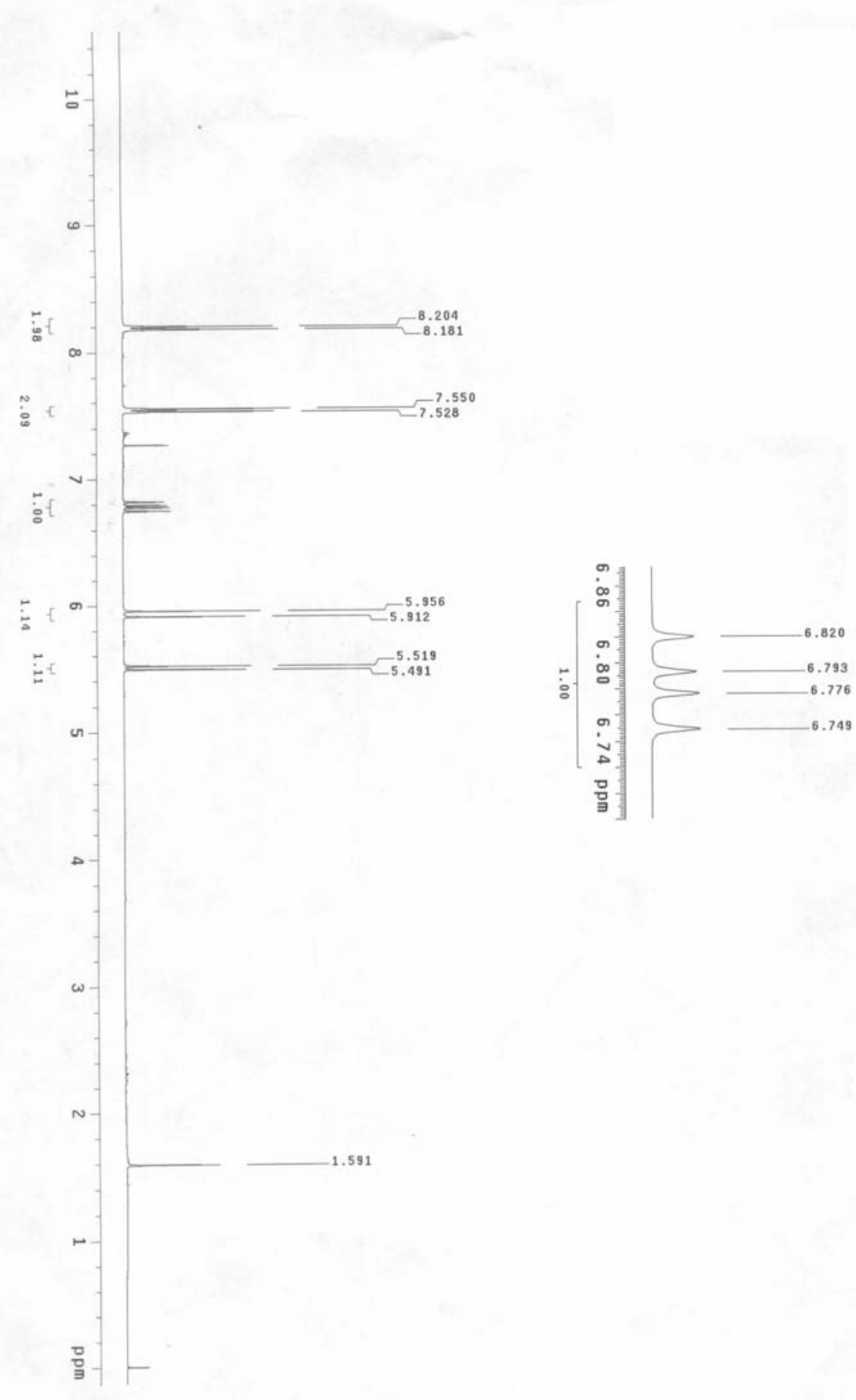
1-Nitro-4-vinylbenzene (4)

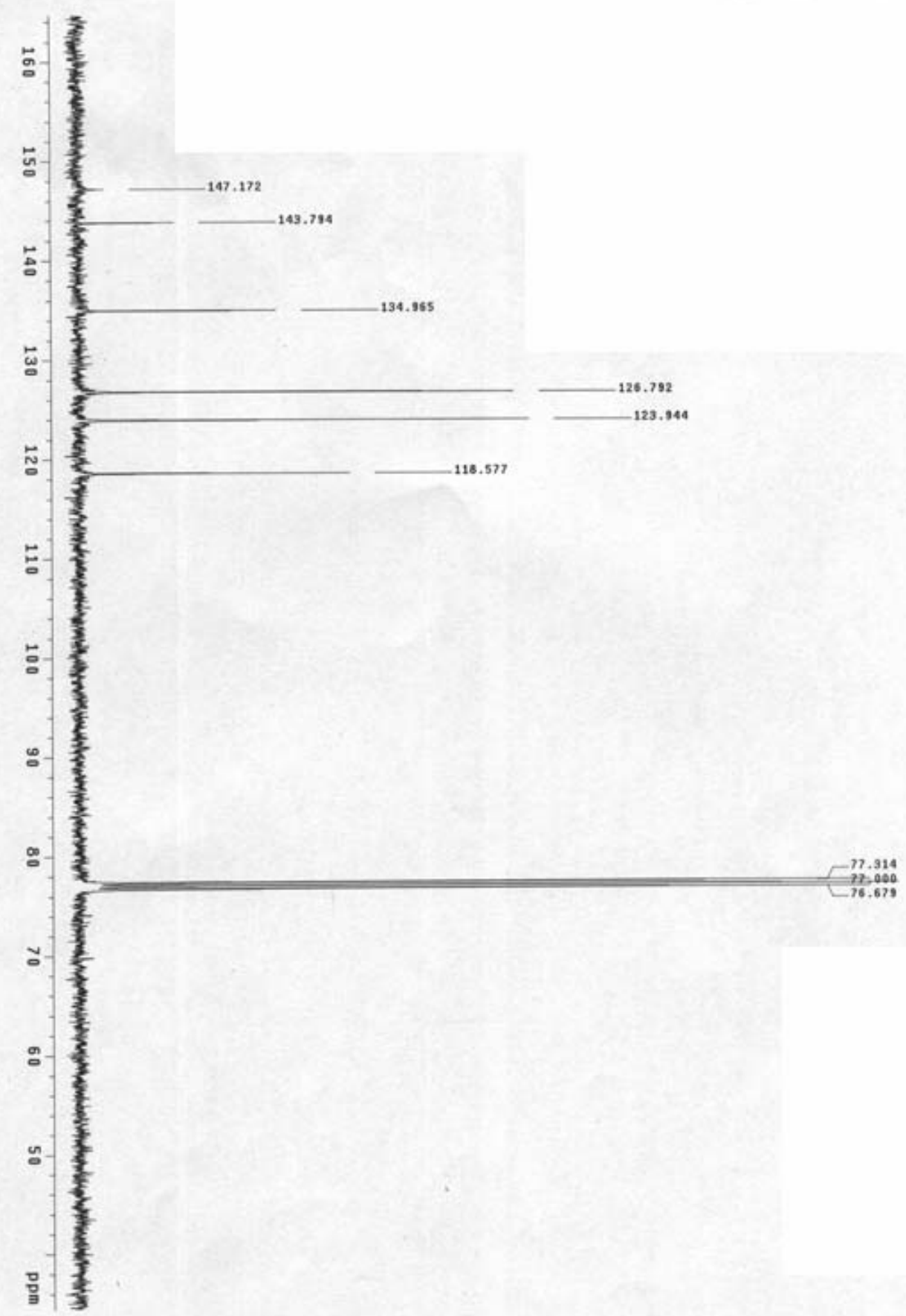


1-(2-(4-Nitrophenyl)ethynyl)benzene (5)
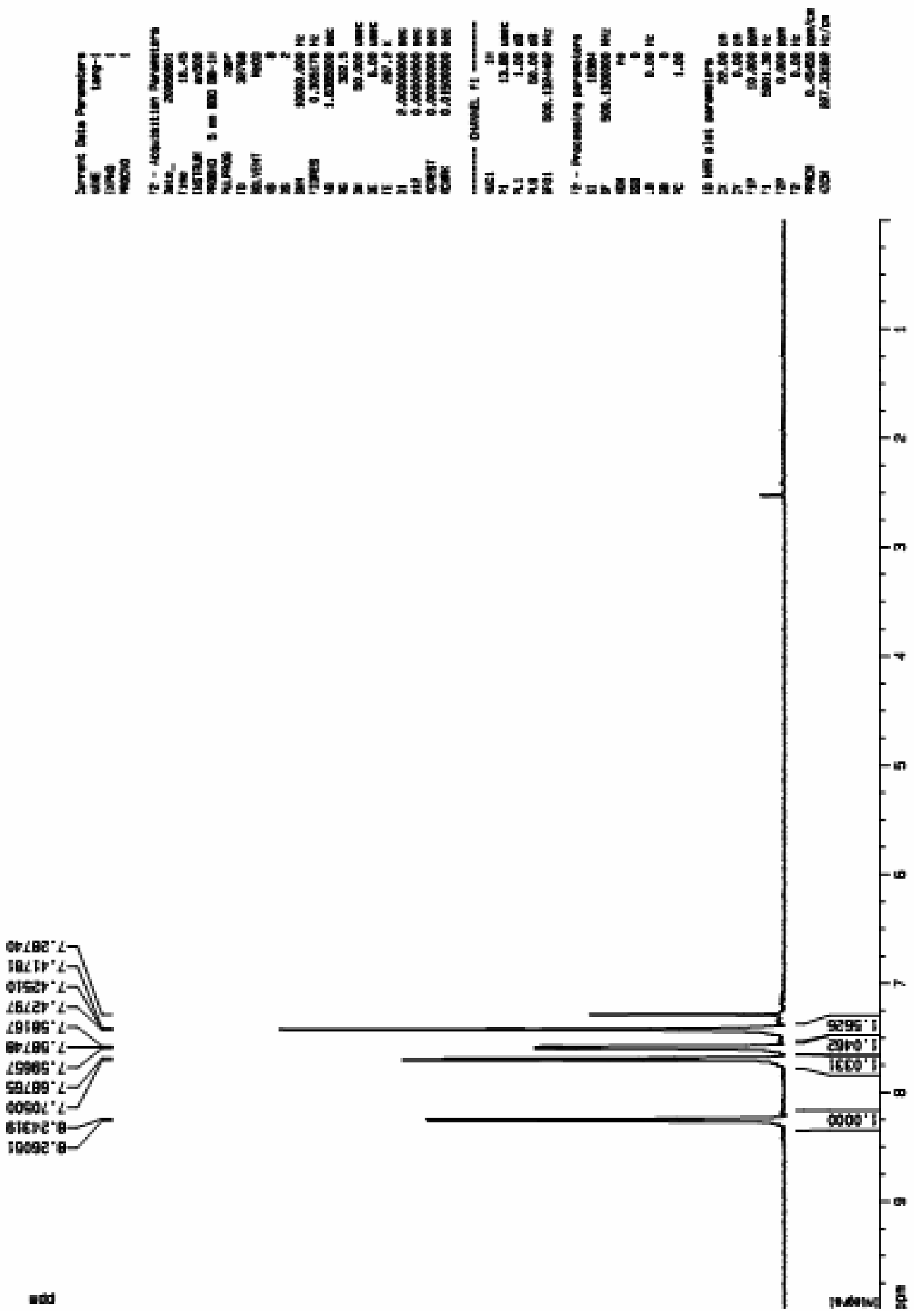
1-(2-(4-Nitrophenyl)ethynyl)benzene (5)
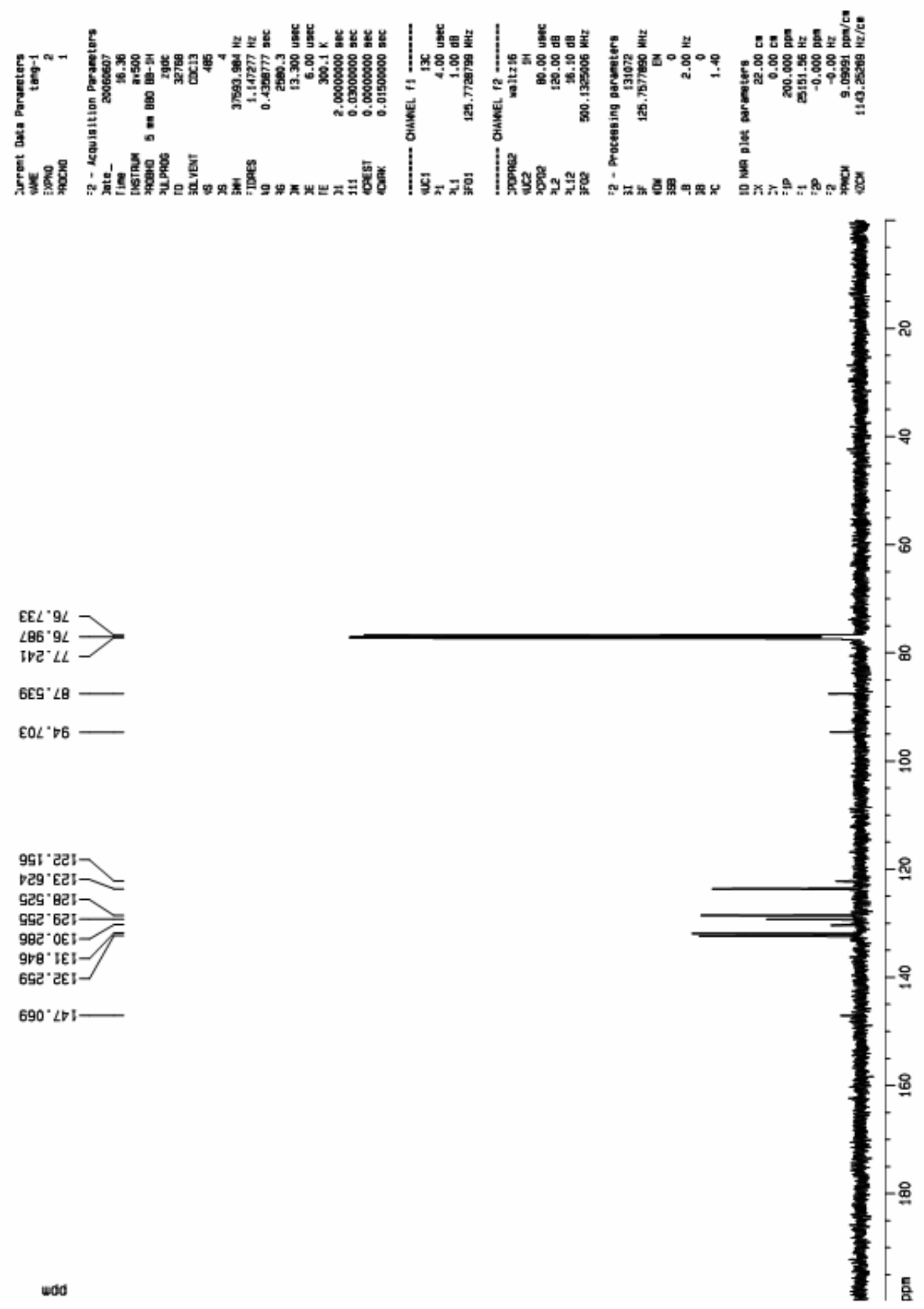
1-Biphenyl-4-yl-ethanone (6)

$1000^{\circ} 0-$

$96 \angle S^{\circ}$

96E9.

$\nabla \angle S C^{\circ} \angle$

$686 \varepsilon<7$

$922 \nabla<$

$\angle 8 \nabla \nabla \cdot \angle]$

$5890^{\circ} \angle 7$

$\angle E \angle \nabla^{\circ} \angle 7$

$0<60 \cdot \angle \neg$

$8019<7$

द9०9 $<-$

$9 \varepsilon \angle 9^{\circ}<-1$

$96 \angle 9^{\circ} \angle-\|$

$8969 \circ-$

$810 L<-$

$0020.8-1$

$5050.8-$

SटV0 8

E8०0.8

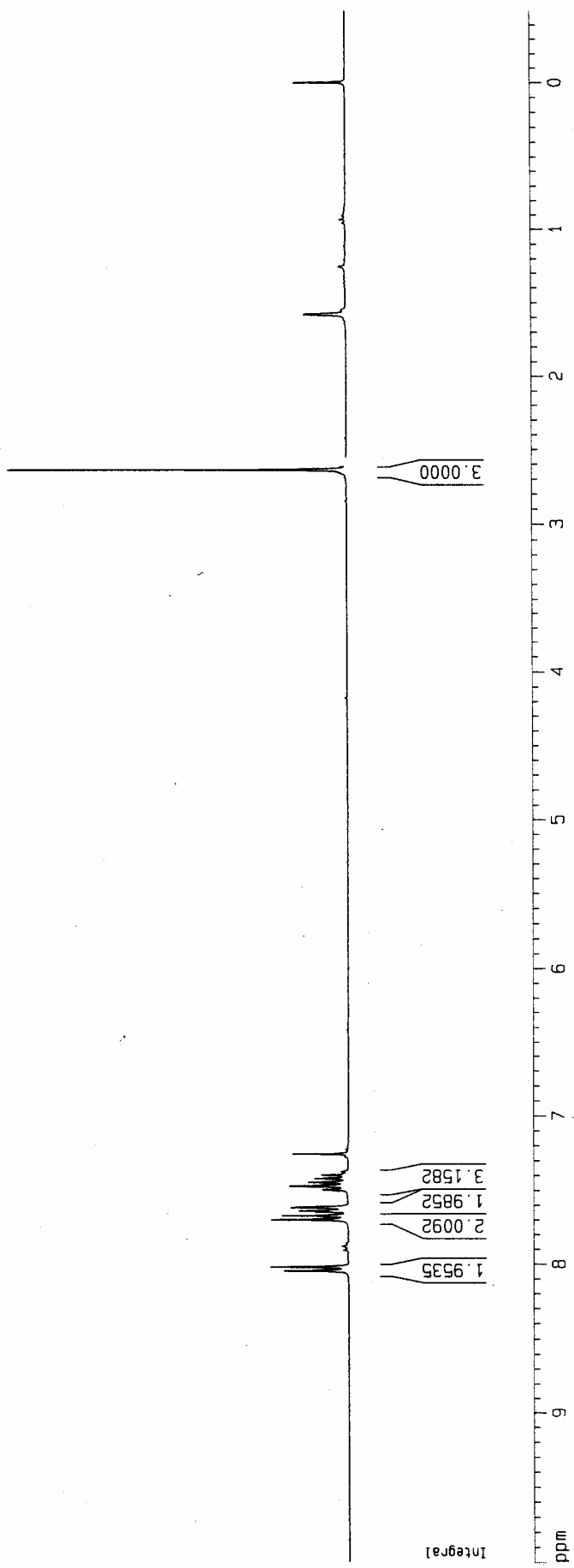


1-Biphenyl-4-yl-ethanone (6)

$996 \cdot 92$

I $66^{\prime} 94$

$\triangle E E^{\prime} \angle L$

$\angle S L L L \longrightarrow$

96081

I09 $\angle 2 โ$

$999 \cdot 8 \mathrm{c}$

घEट ' 62

ट90 OE -

8 I $9 E$

gटट O० [-

IEI. 901

$990^{\circ} 85$

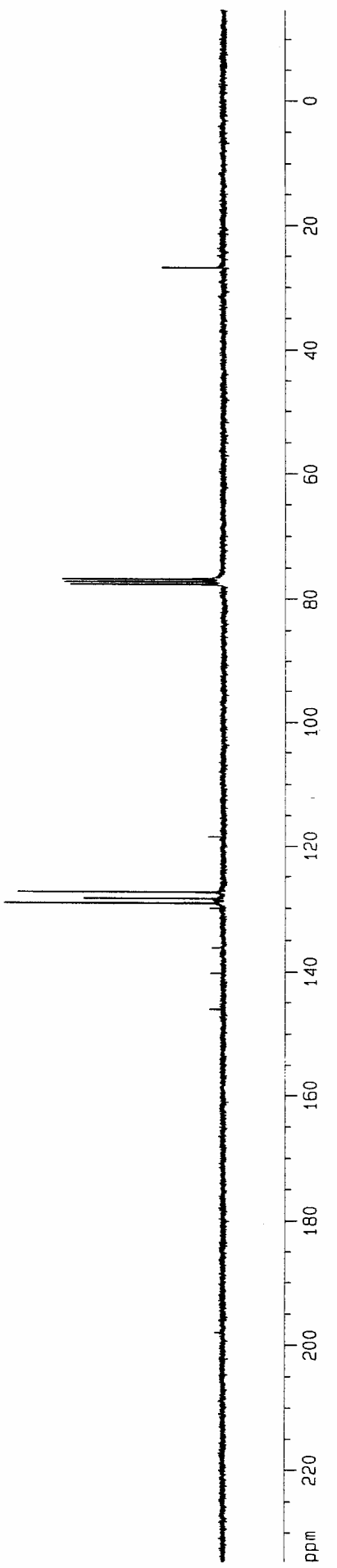


4-Methoxy-biphenyl (7)
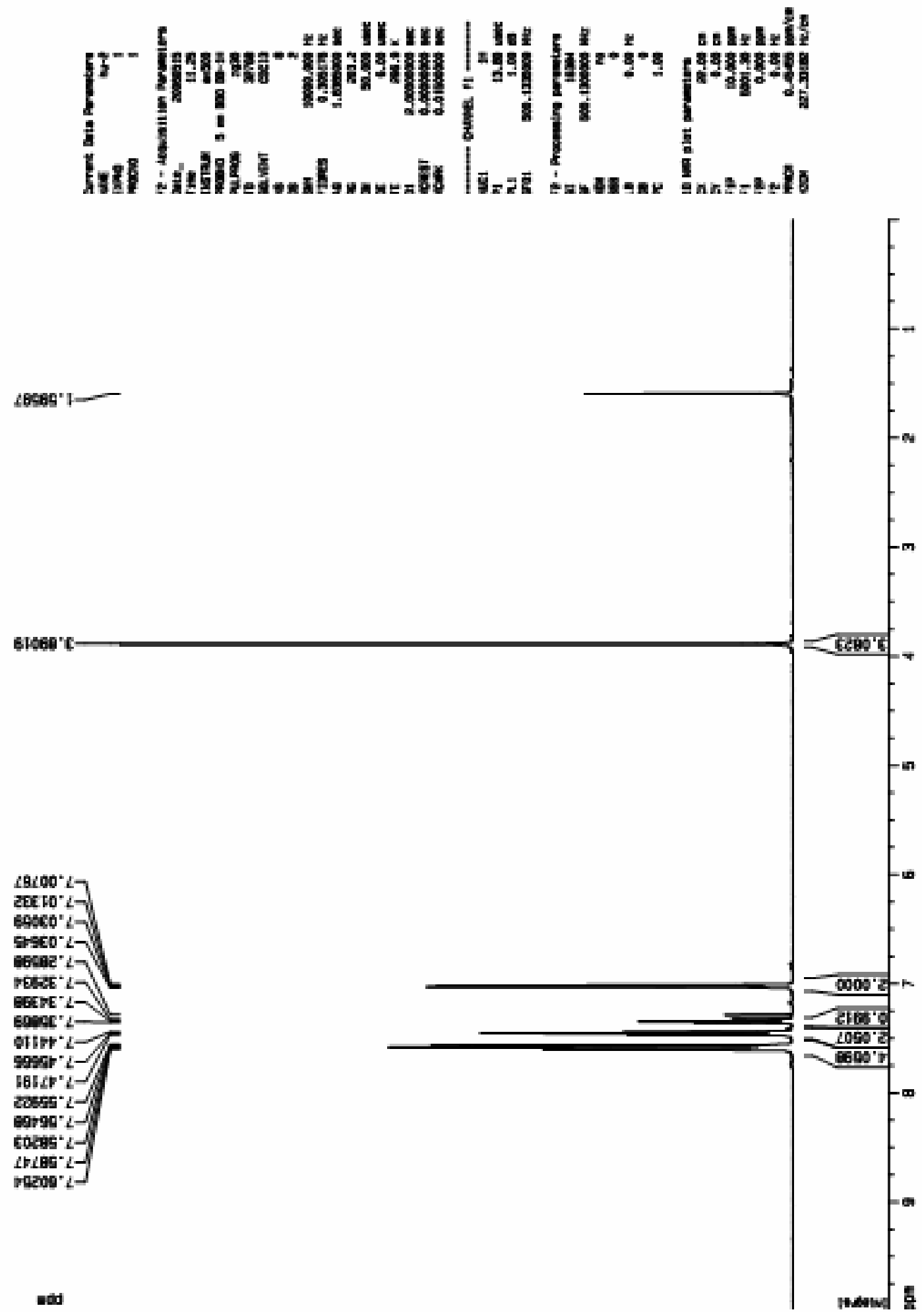
4-Methoxy-biphenyl (7)

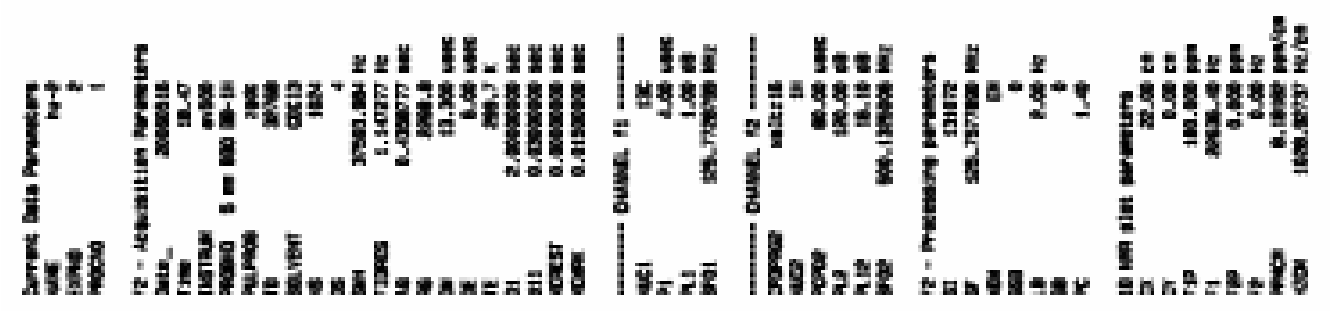

$\cos 2=$

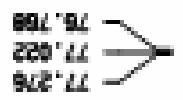

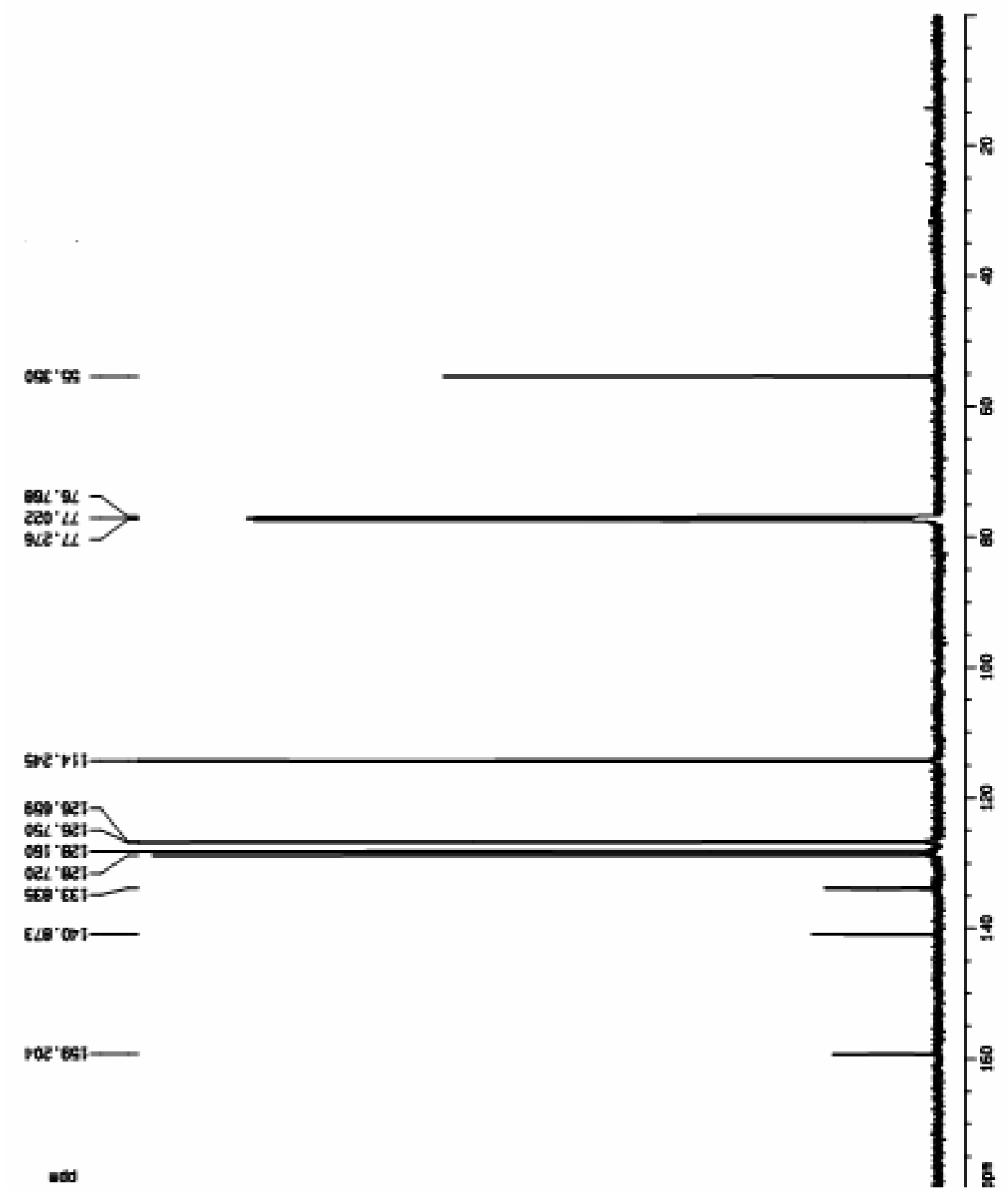


1-(2-(4-Methoxyphenyl)ethynyl)benzene (8)

$20000^{\circ} 0$

2ESDS:-

EDBC8 $E$

$90 \angle 98^{\circ} 9$

1688.9

O65SE L

ODSEE : $\angle 7$

$\nabla 2090^{\circ}<7$

$\varepsilon D 580^{\circ} L$

$\triangle 6860^{\circ}:-$

$6 \angle 20 S^{\circ}<-$

$96905^{\circ} L$

IBgIS' $L-$

$16 \angle 15^{\circ}<-$

9V2टद $\angle-$

udd

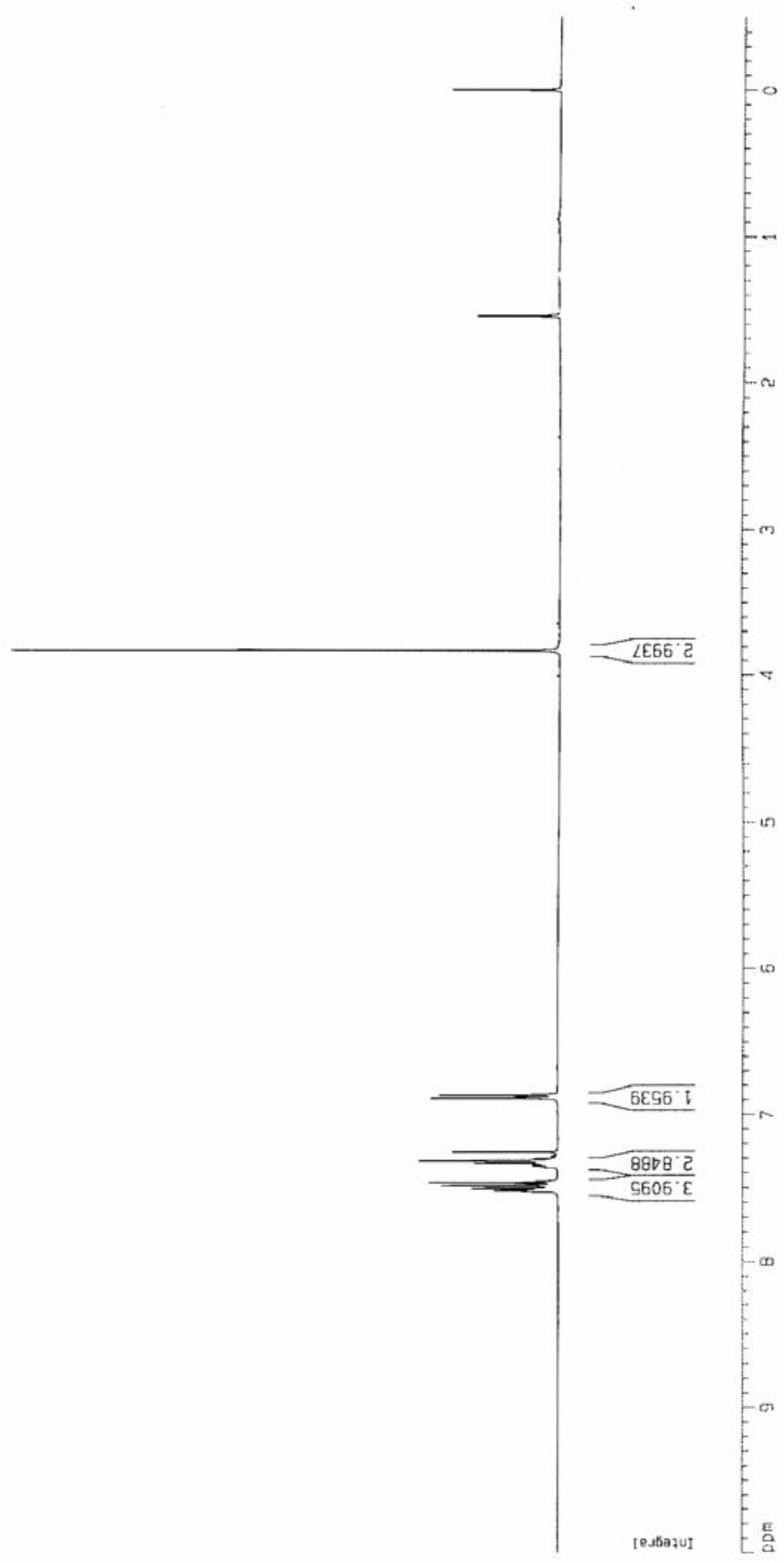


1-(2-(4-Methoxyphenyl)ethynyl)benzene (8)
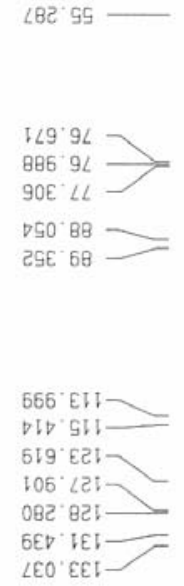

2E9 $695-$

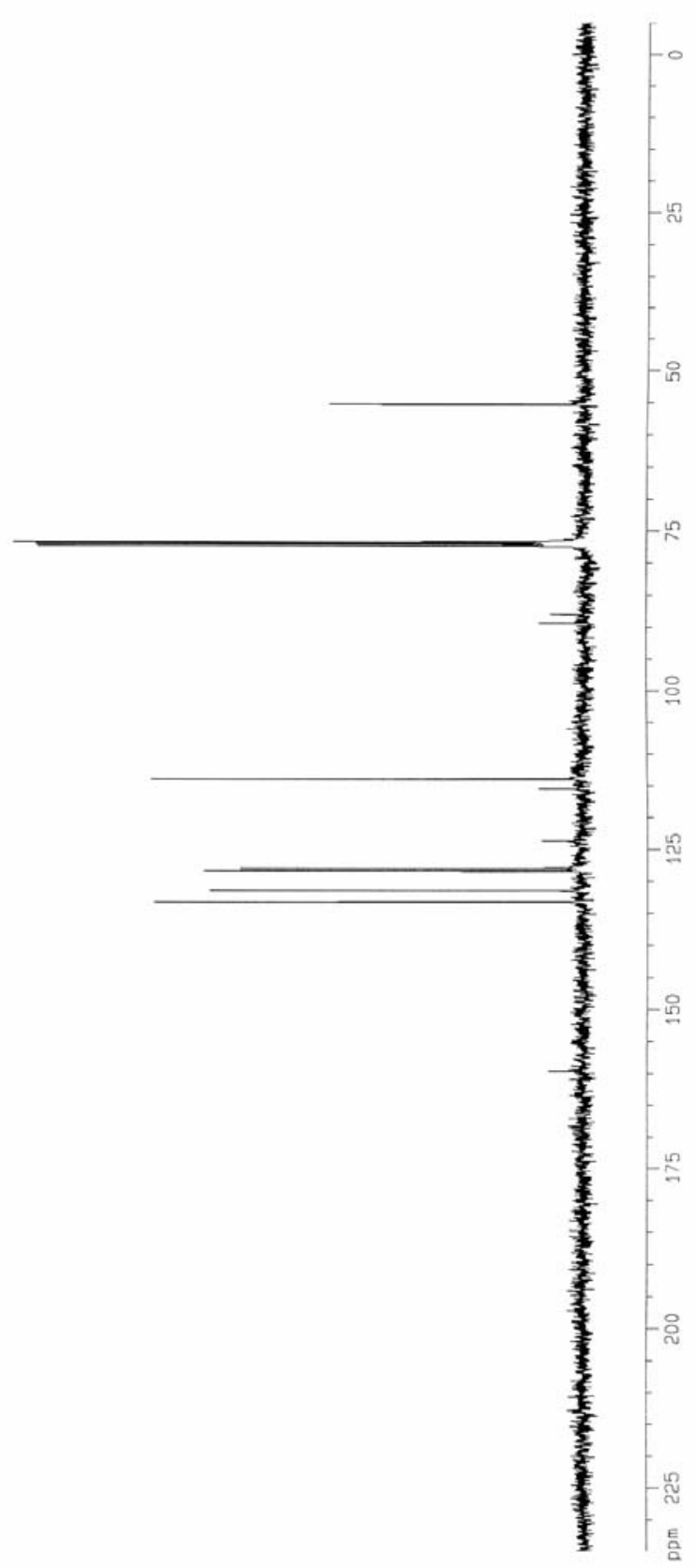


1-(4-(2-Phenylethynyl)phenyl)ethanone (9)

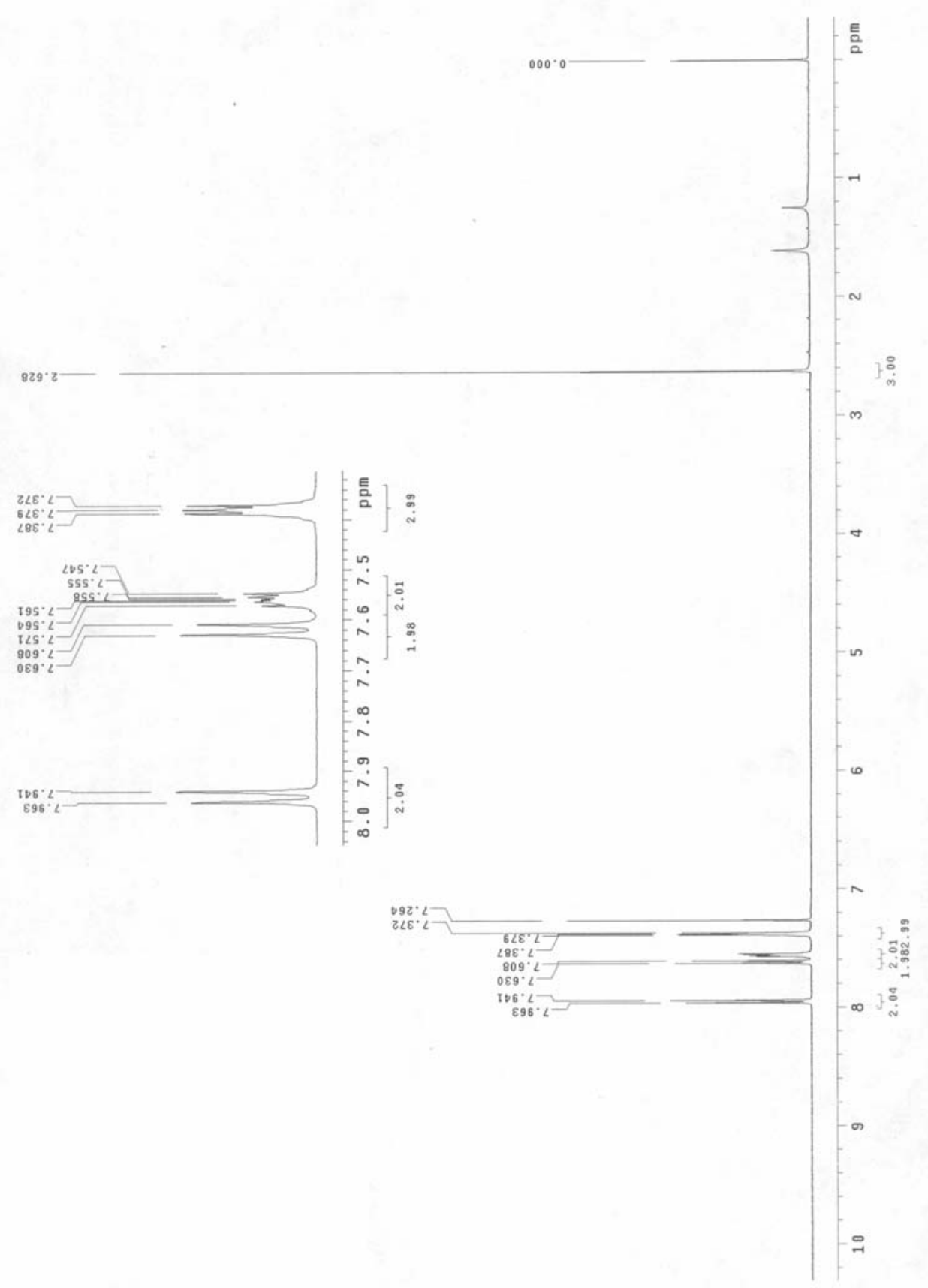


1-(4-(2-Phenylethynyl)phenyl)ethanone (9)

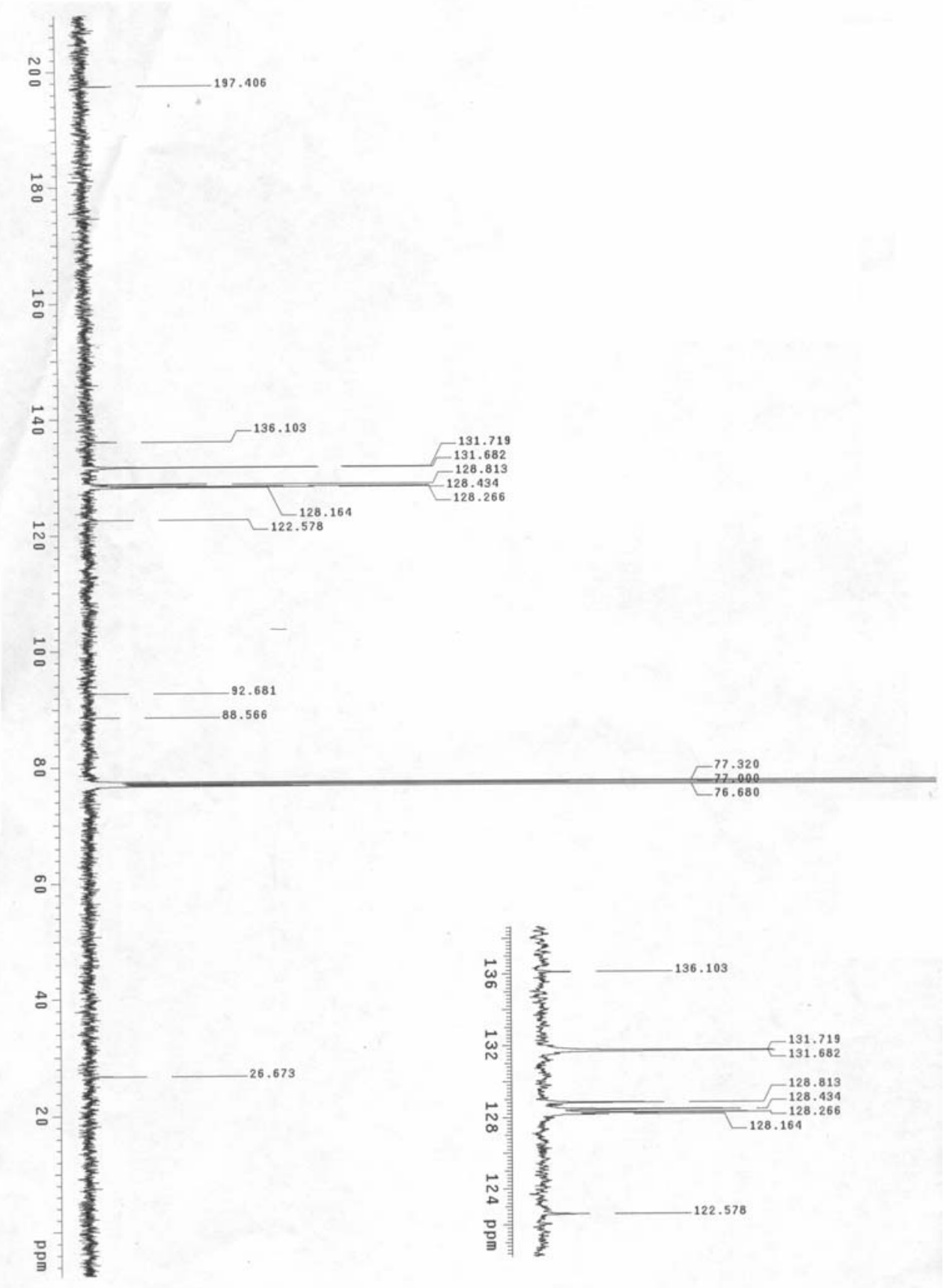


Biphenyl (10)

$1000^{\circ} 0-$

DIIG

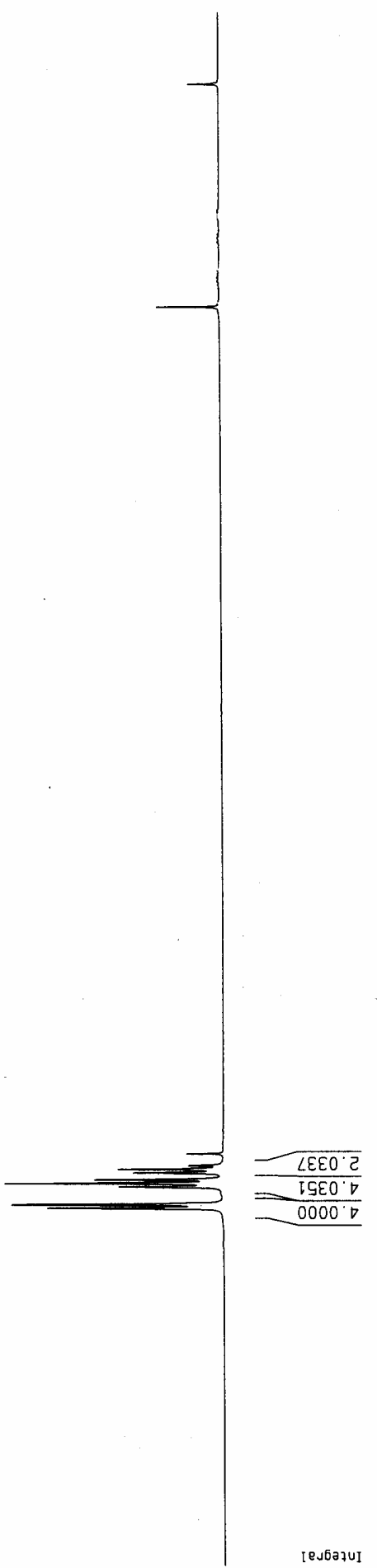

टटाह $<7$

ट6टह $L]$

99EE $L \neg$

8EDE $L \neg$

999E $L>$

$809 E<$

6D9E $L$

$8800^{\circ} L$

96टt $\angle-F$

$\triangle \nabla E D<-$

$\nabla ट S \circ<1$

[85 $\angle]$

टR $\mathrm{G}<-$

$6969^{\circ}<$

โ209 $\angle$

$6909^{\circ} \mathrm{L}$ 
Biphenyl (10)
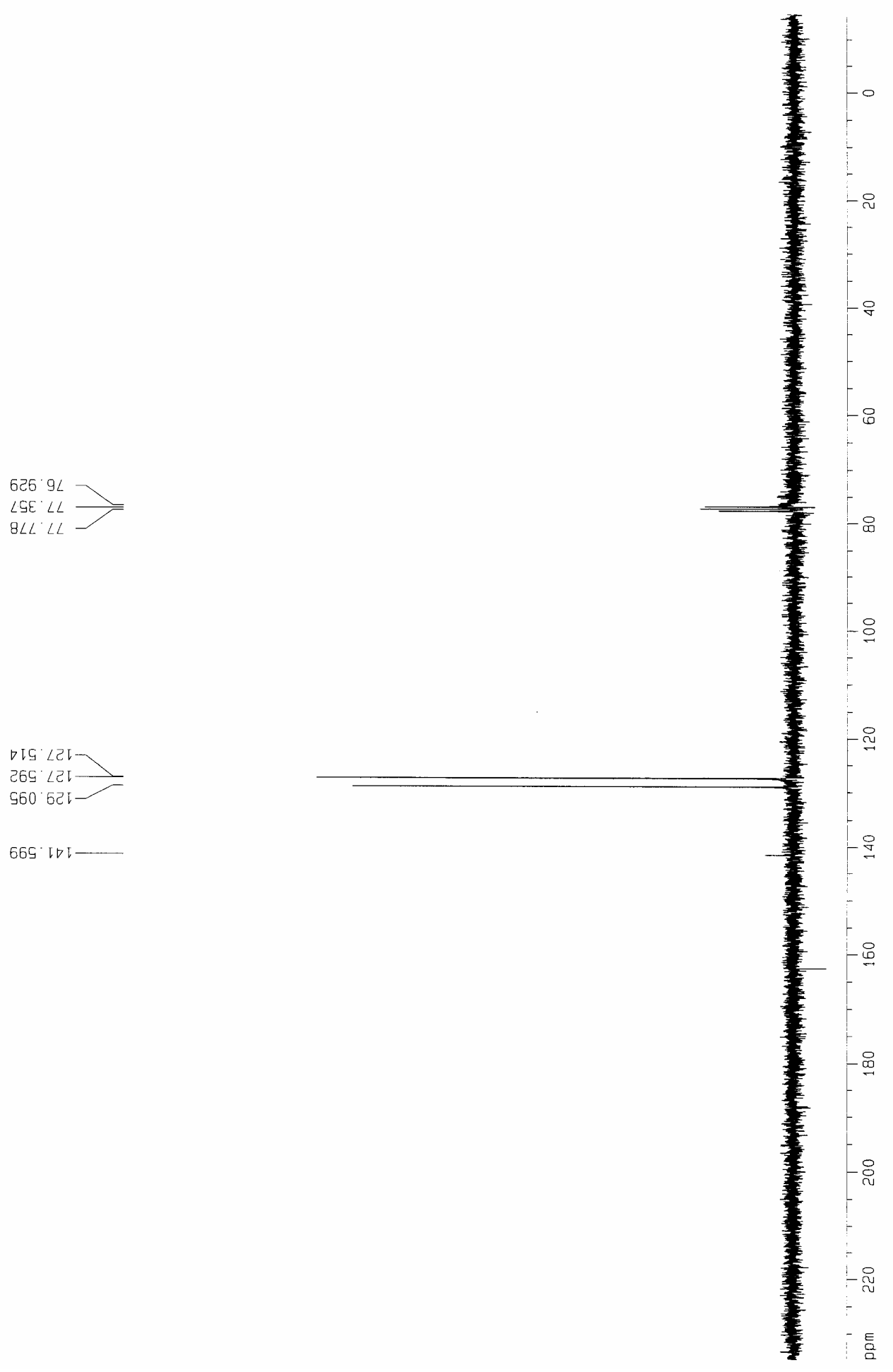
1,2-Diphenylethyn (11)
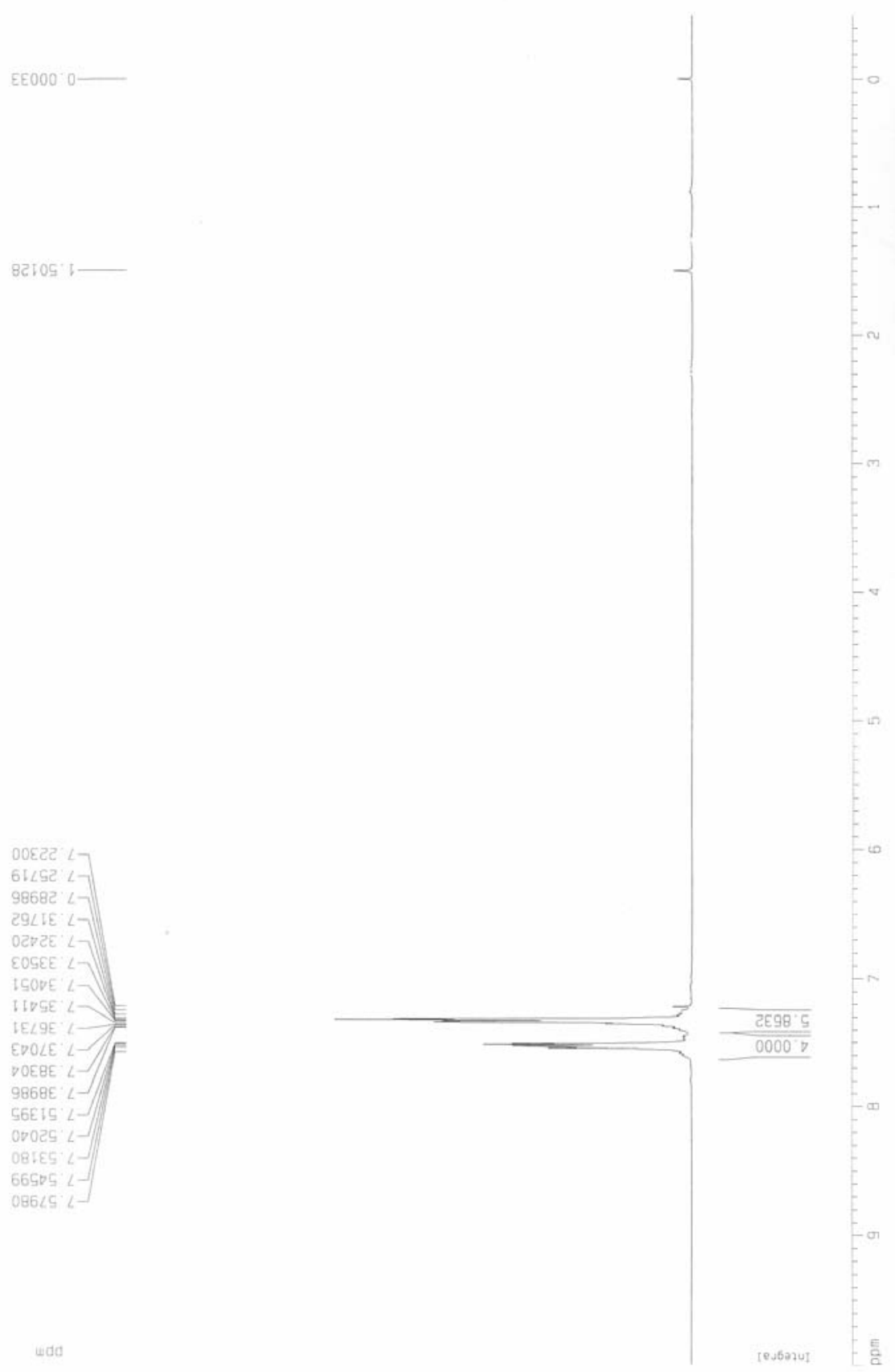
1,2-Diphenylethyn (11)
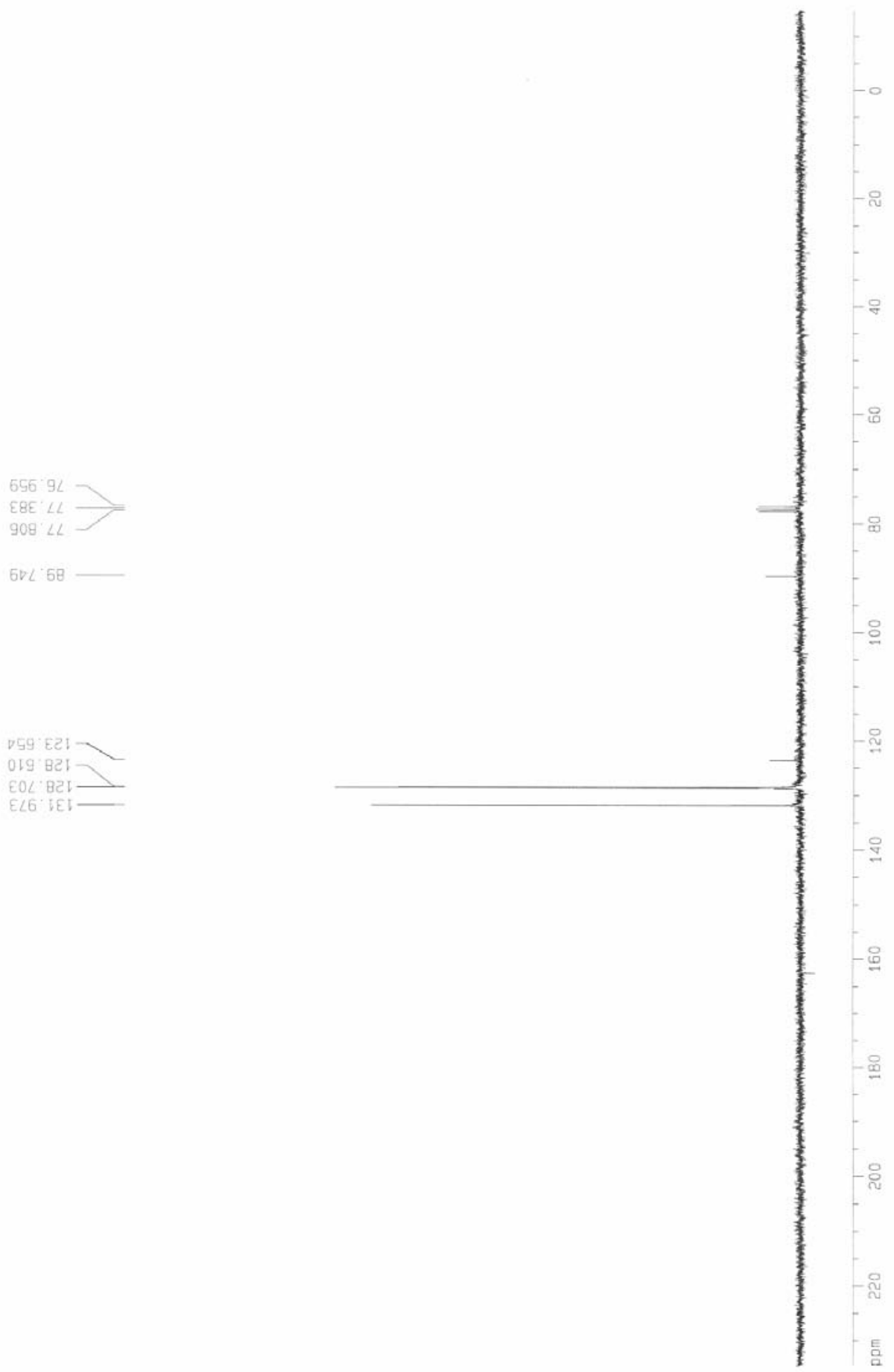
4-Methyl-biphenyl (12)

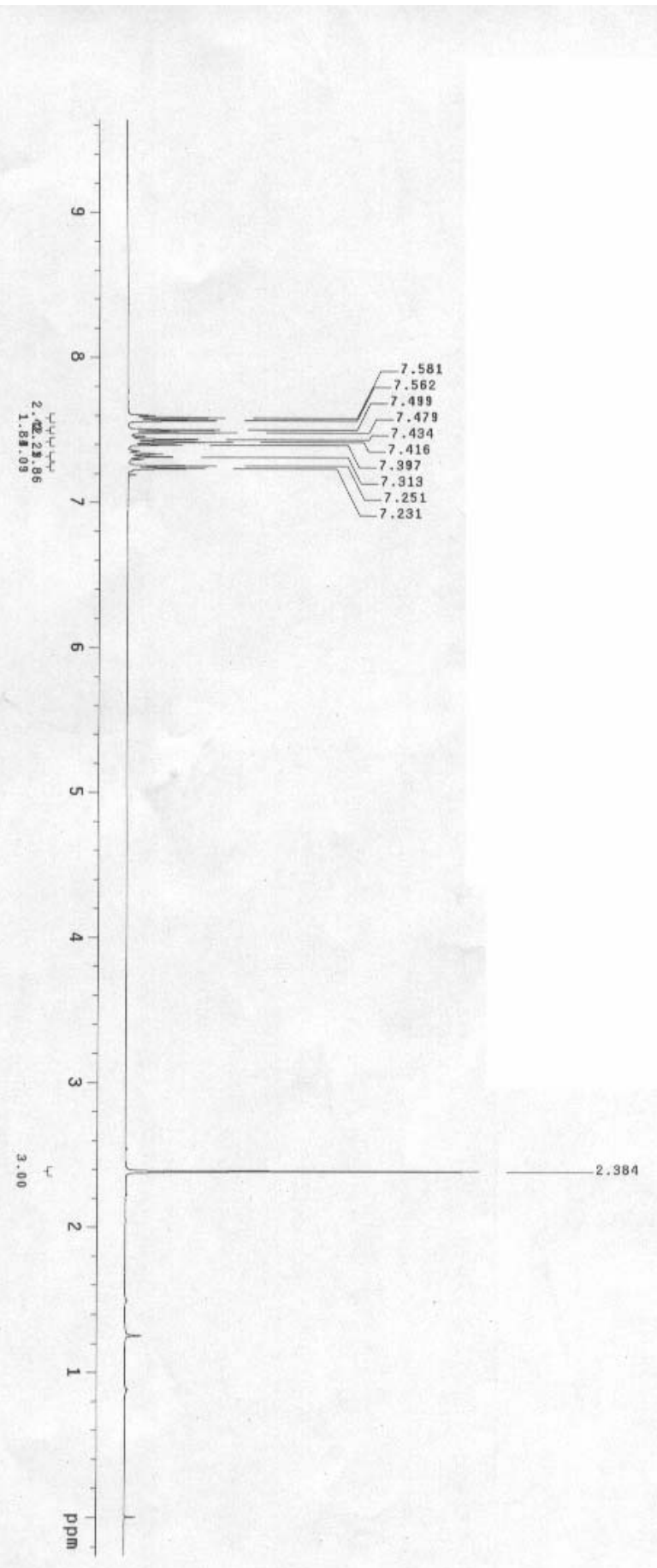


4-Methyl-biphenyl (12)

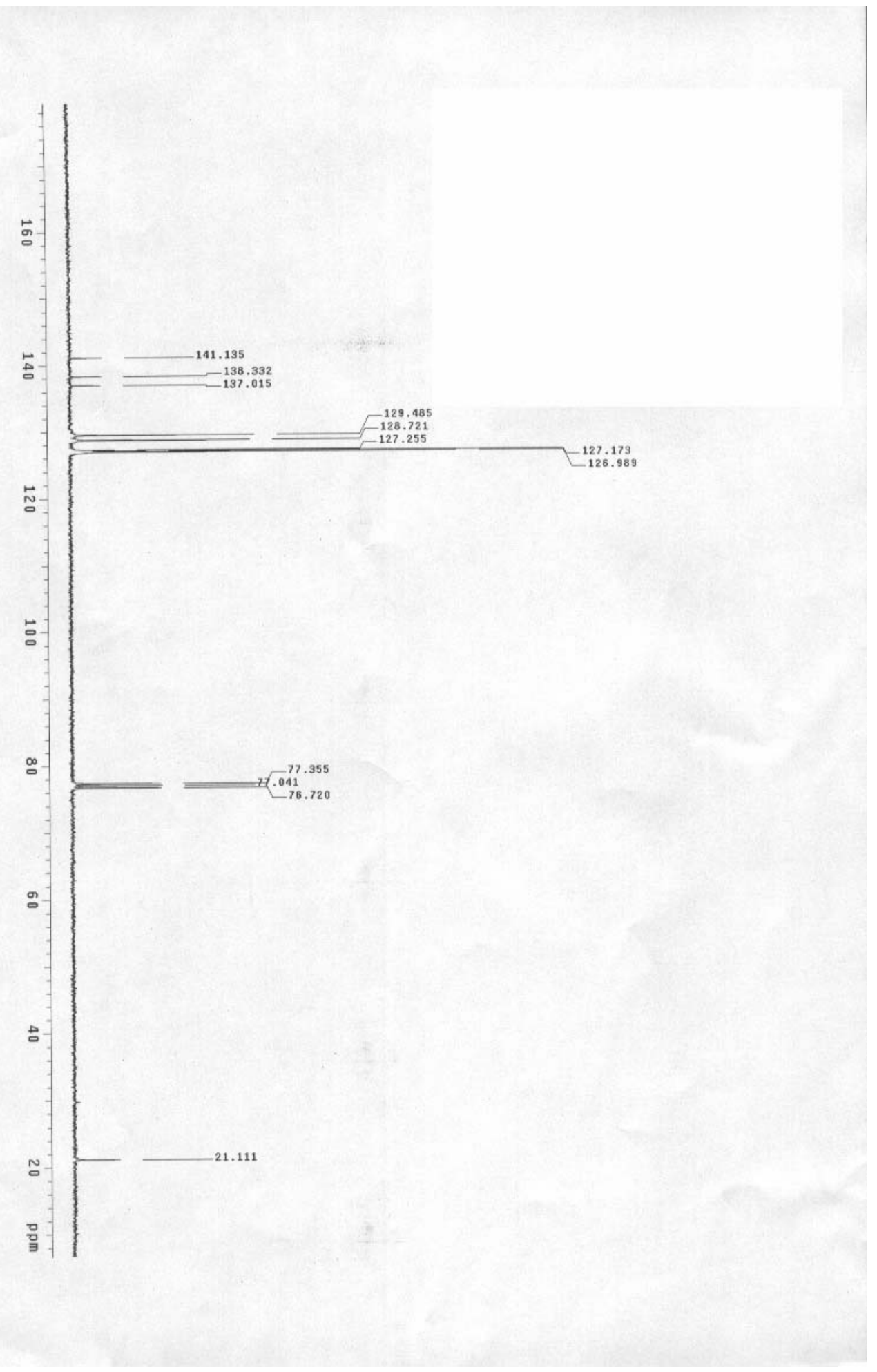




\section{3,5-Dimethyl-biphenyl (13)}
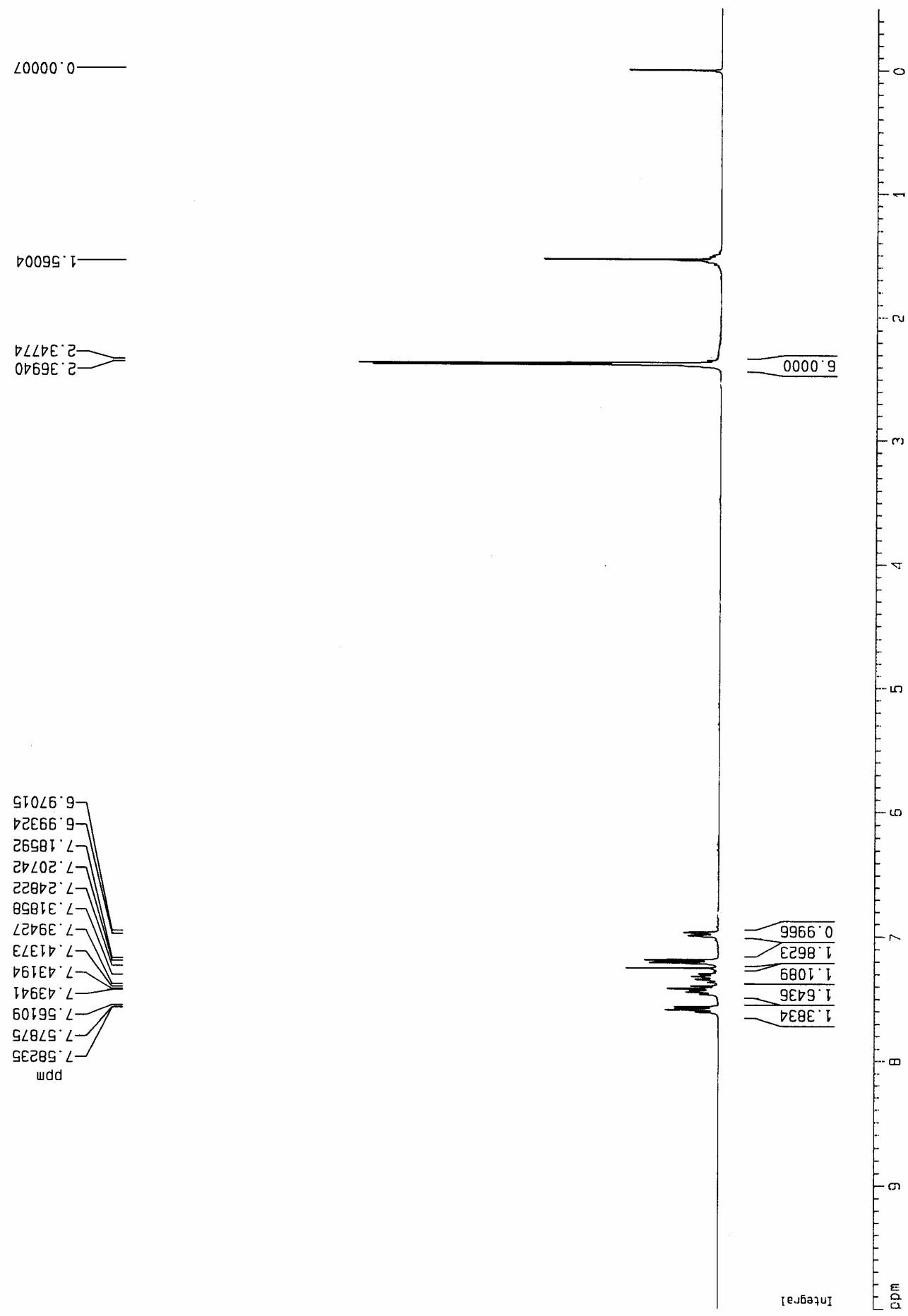
3,5-Dimethyl-biphenyl (13)

GLE' IC

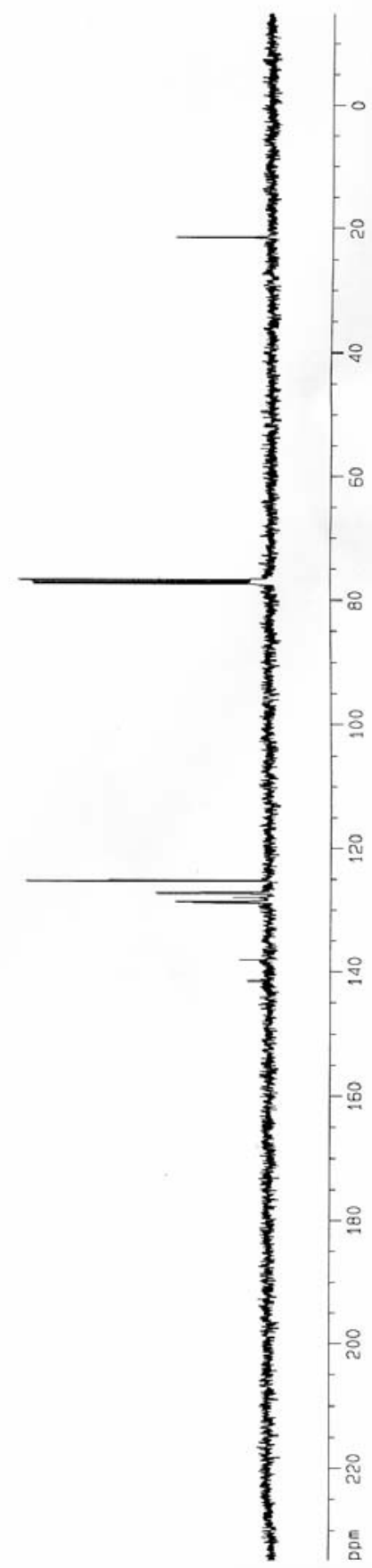

